MATHEMATICS OF COMPUTATION

Volume 76, Number 257, January 2007, Pages 67-96

S 0025-5718(06)01895-3

Article electronically published on August 7, 2006

\title{
SUPERCONVERGENCE OF THE NUMERICAL TRACES OF DISCONTINUOUS GALERKIN AND HYBRIDIZED METHODS FOR CONVECTION-DIFFUSION PROBLEMS IN ONE SPACE DIMENSION
}

\author{
FATIH CELIKER AND BERNARDO COCKBURN
}

\begin{abstract}
In this paper, we uncover and study a new superconvergence property of a large class of finite element methods for one-dimensional convectiondiffusion problems. This class includes discontinuous Galerkin methods defined in terms of numerical traces, discontinuous Petrov-Galerkin methods and hybridized mixed methods. We prove that the so-called numerical traces of both variables superconverge at all the nodes of the mesh, provided that the traces are conservative, that is, provided they are single-valued. In particular, for a local discontinuous Galerkin method, we show that the superconvergence is order $2 p+1$ when polynomials of degree at most $p$ are used. Extensive numerical results verifying our theoretical results are displayed.
\end{abstract}

\section{INTRODUCTION}

In this paper, we obtain a new superconvergence result for a large class of finite element methods for one-dimensional convection-diffusion problems. This class includes discontinuous Galerkin (DG) methods devised by means of numerical traces, discontinuous Petrov-Galerkin methods, and hybridized mixed methods. We work in the framework of the model problem

$$
\begin{array}{ll}
-\epsilon u^{\prime \prime}+c u^{\prime}=f & \text { in } \Omega=(0,1), \\
u=u_{D} & \text { on } \partial \Omega=\{0,1\},
\end{array}
$$

where the velocity $c$ is a nonnegative constant and the diffusion coefficient $\epsilon$ a positive real number. We chose this problem only for the sake of clarity and simplicity. All the results we obtain can be easily extended to more general convection-diffusion problems.

Let us briefly describe our result. We consider methods that provide an approximation $\left(q_{h}, u_{h}\right)$ to the solution $(q, u)$ of

$$
\begin{aligned}
q & =\epsilon u^{\prime} & & \text { in } \Omega, \\
-(q-c u)^{\prime} & =f & & \text { in } \Omega, \\
u & =u_{D} & & \text { on } \partial \Omega .
\end{aligned}
$$

Received by the editor May 12, 2005.

2000 Mathematics Subject Classification. Primary 65M60, 65N30, 35L65.

The second author was partially supported by the National Science Foundation (Grant DMS0411254) and by the Minnesota Supercomputing Institute. 
The methods we consider rely on a suitable definition of the so-called numerical traces $\widehat{u}_{h}^{\epsilon}$ and $\left(-\widehat{q}_{h}+c \widehat{u}_{h}^{c}\right)$ which are nothing but approximations to the potential $u$ and to its flux $(-q+c u)$, respectively. We show that, if they are conservative, that is, if they are single-valued, these two numerical traces superconverge at the nodes of the mesh. In particular, if polynomials of degree at most $p$ are used for the approximation $\left(q_{h}, u_{h}\right)$ given by a suitably defined LDG method, the superconvergence is of order $2 p+1$. We also show that in the purely convective case, that is, if $\epsilon=0$, the numerical trace of the flux is always exact; and that, in the absence of convection, that is, when $c=0$, both numerical traces are always exact.

Let us contrast this result with similar superconvergence results in the available literature. First, let us consider the purely convective case, namely, the case when $\epsilon=0$. In the first analysis of the original DG method [23], Lesaint and Raviart also considered the DG method applied to a simple ODE. They showed that a collocation version of the DG method using the upwinding numerical flux was nothing but an implicit Runge-Kutta method of order $2 p+1$ when polynomials of degree $p$ are used. Later, Delfour et al. [19] proved that the numerical trace of the DG method superconverges at the nodes with order $2 p+1$ for a fairly general class of numerical traces. These results imply ours for DG methods with conservative numerical traces.

Now, let us consider the purely elliptic case, that is, the case in which $c=0$. It is very well known that in this case, the values at the nodes of the approximation to the potential given by the classical $H^{1}$-conforming finite element method are exact. A similar result holds for DG methods. Indeed, in [13] it was proven that if both numerical traces are conservative and consistent, the numerical trace of the potential is exact. On the other hand, in [22] Larson and Niklasson showed that if uniform meshes are used, the numerical trace for the flux is also exact at the nodes for the interior penalty (IP) method, and of order $p$ for the nonsymmetric interior penalty Galerkin (NIPG) method. Our main theorem implies all the abovementioned results. Note, in particular, that, unlike the IP method, the numerical trace of the flux of the B.O. method cannot be exact because its numerical trace for the potential is not conservative.

Finally, let us consider the convection-diffusion case. In [20], Douglas and Dupont proved that, when applied to general convection-diffusion equations, the method superconverges at the nodes of the mesh with order $2 p$ when polynomials of degree at most $p$ are used. In [28], J. Wheeler proposed a postprocessing that allowed to compute approximations to the flux at the boundary of the computational domain. In [29], the procedure was extended for the computation of approximations to the flux at all the nodes and a superconvergence of order $2 p$ was proven therein. For a unified theory of superconvergence for continuous Galerkin methods, see Dupont [21]. Our results imply that DG methods employing polynomial approximations of degree $p$ have both numerical traces super-converging with order $2 p+1$ for some suitably chosen DG methods. Just as proposed in [20], by using a simple and local postprocessing, we can obtain approximations for both the potential $u$ and its flux $(-q+c u)$ that converge uniformly with order $2 p+1$ in the whole domain.

In this paper, we have not dealt with two important superconvergence properties. The first is the identification of superconvergence points of the approximation inside each element; see the recent work on superconvergence for DG methods [1] and the references therein. The other property, the uniformity of the superconvergence as 
the parameter $\epsilon$ goes to zero, will be considered elsewhere. It requires a different type of analysis and the use of Shishkin-like meshes to properly deal with the boundary layers; see the recent work in [30] and [31].

The organization of the rest of the paper is as follows. In Section 2, we describe the DG methods under consideration, state our main result, Theorem 2.1, and discuss some of its consequences; we also present a variation of this result for a special LDG method, Theorem 2.6. These theorems are proven in Section 3. In Section 4, we display numerical experiments that verify our theoretical findings. Finally, in Section 5, we end by pointing out some straightforward extensions and giving some concluding remarks.

\section{The MAIN RESUlT}

In this section, we describe the class of methods under consideration, state our main result, and discuss some of its consequences.

2.1. The general form of the methods. To describe the structure of the methods under consideration, let us begin by partitioning the domain $\Omega=(0,1)$. Thus, if $0=x_{0}<x_{1}<\cdots<x_{N-1}<x_{N}=1$, the partition is $\Omega_{h}=\left\{I_{j}=\left(x_{j-1}, x_{j}\right), j=\right.$ $1, \ldots, N\}$. For each interval $I_{j} \in \Omega_{h}$, we define its outward unit normal $n_{I_{j}}\left(x_{j}\right)=1$ and $n_{I_{j}}\left(x_{j-1}\right)=-1$; if there is no confusion, instead of $n_{I_{j}}$ we simply write $n$.

Next, we describe the weak formulation used by the methods. Such a formulation is satisfied by the exact solution and is obtained as follows. If the restriction of the exact solution $(q, u)$ to the interval $I_{j}$ belongs to $H^{1}\left(I_{j}\right) \times H^{1}\left(I_{j}\right)$, we immediately have

$$
\begin{aligned}
& (q, v)_{I_{j}}=-\left(\epsilon u, v^{\prime}\right)_{I_{j}}+\langle\epsilon u, v n\rangle_{\partial I_{j}}, \\
& \left(q-c u, w^{\prime}\right)_{I_{j}}-\langle q-c u, w n\rangle_{\partial I_{j}}=(f, w)_{I_{j}},
\end{aligned}
$$

for all $(v, w) \in H^{1}\left(I_{j}\right) \times H^{1}\left(I_{j}\right)$. Here, we have used the notation

$$
(\varphi, \psi)_{I_{j}}:=\int_{I_{j}} \varphi(x) \psi(x) d x
$$

and

$$
\langle\varphi, \psi n\rangle_{\partial I_{j}}=\varphi\left(x_{j}^{-}\right) \psi\left(x_{j}^{-}\right)-\varphi\left(x_{j-1}^{+}\right) \psi\left(x_{j-1}^{+}\right) .
$$

As a consequence, if $(q, u) \in H^{1}\left(\Omega_{h}\right) \times H^{1}\left(\Omega_{h}\right)$, we have

$$
\begin{aligned}
& (q, v)_{\Omega_{h}}=-\left(\epsilon u, v^{\prime}\right)_{\Omega_{h}}+\langle\epsilon u, v n\rangle_{\partial \Omega_{h}}, \\
& \left(q-c u, w^{\prime}\right)_{\Omega_{h}}-\langle q-c u, w n\rangle_{\partial \Omega_{h}}=(f, w)_{\Omega_{h}}, \\
& \left.u\right|_{\partial \Omega}=u_{D},
\end{aligned}
$$

where

$$
(\varphi, \psi)_{\Omega_{h}}:=\sum_{I_{j} \in \Omega_{h}}(\varphi, \psi)_{I_{j}} \quad \text { and } \quad\langle\varphi, \psi n\rangle_{\partial \Omega_{h}}:=\sum_{I_{j} \in \Omega_{h}}\langle\varphi, \psi n\rangle_{\partial I_{j}} .
$$

The approximate solution $\left(q_{h}, u_{h}\right)$ given by the methods under consideration satisfies a similar weak formulation. Indeed, it is sought in a finite-dimensional subspace 
of $H^{1}\left(\Omega_{h}\right) \times H^{1}\left(\Omega_{h}\right), V_{h} \times W_{h}$, and is required to satisfy

$$
\begin{aligned}
& \left(q_{h}, v\right)_{\Omega_{h}}=-\left(\epsilon u_{h}, v^{\prime}\right)_{\Omega_{h}}+\left\langle\epsilon \widehat{u}_{h}^{\epsilon}, v n\right\rangle_{\partial \Omega_{h}}, \\
& \left(q_{h}-c u_{h}, w^{\prime}\right)_{\Omega_{h}}-\left\langle\widehat{q}_{h}-c \widehat{u}_{h}^{c}, w n\right\rangle_{\partial \Omega_{h}}=(f, w)_{\Omega_{h}}, \\
& \left.\widehat{u}_{h}^{\epsilon}\right|_{\partial \Omega}=u_{D},
\end{aligned}
$$

for all $(v, w) \in \mathbb{V}_{h} \times \mathbb{W}_{h}$.

To complete the definition of the method, it remains to define the trial and test spaces, $V_{h} \times W_{h}$ and $\mathbb{V}_{h} \times \mathbb{W}_{h}$, as well as the numerical trace for the potential, $\widehat{u}_{h}^{\epsilon}$, and for its flux, $-\widehat{q}_{h}+c \widehat{u}_{h}^{c}$, at the nodes of the mesh, $\mathscr{E}_{h}=\left\{x_{i}\right\}_{i=0}^{N}$. The precise definition of the above-mentioned spaces and of the numerical traces, however, is irrelevant for our main result since it is completely independent of them. Indeed, it applies to any well-defined method whose approximate solution satisfies the abovedescribed weak formulation.

2.2. Examples. Let us display some examples of such methods. In Tables 1, 2 and 3, extracted mostly from [4], we display the the numerical traces and the space of some methods satisfying (2.2).

We divide them in three groups. The four methods of the first group have numerical fluxes $\widehat{u}_{h}^{\epsilon}$ that are not conservative. All the methods of the second group are DG methods whose numerical fluxes are conservative. The two methods of the last group are the hybridized Raviart-Thomas method [24, 3], denoted here by h-R.T., and the discontinuous Petrov-Galerkin method [10], denoted here by DPG.

Almost all the methods of the first two groups have been considered in the unifying analysis of DG methods proposed in [4]. There are three exceptions. The first, called the modified Babuška-Zlámal method and denoted by m-B.Z., has both numerical fluxes conservative; note that the original method of Babuška and Zlámal [5] does not have a conservative numerical trace $\widehat{u}_{h}^{\epsilon}$. The other two are what we could call the minimal dissipation methods since they do not penalize the jumps of the potential at the interelement boundaries. For this reason, we denote them by md-LDG $\left(\left.\gamma\right|_{\mathscr{E}_{h}^{\circ}}=0\right)$ and md-DG $\left(\left.\gamma\right|_{\mathscr{E}_{h}^{\circ}} \neq 0\right)$. They were introduced in [18]; the md-LDG method was further studied in [14].

The methods of the third group have the property that some or all of their numerical traces are new unknowns. Note that the h-R.T. method requires the additional equations

$$
q_{h}\left(x_{j}^{-}\right)=q_{h}\left(x_{j}^{+}\right), \quad j=1, \ldots, N-1,
$$

to be well defined.

In Table 1, we use the following notation to describe the numerical traces at the interior nodes. The average of the trace of $\varphi$ at the interior node $x_{j}$ is given by

$$
\{\varphi\}\}\left(x_{j}\right):=\frac{1}{2}\left(\varphi\left(x_{j}^{+}\right)+\varphi\left(x_{j}^{-}\right)\right),
$$

and its jump by

$$
\llbracket \varphi n \rrbracket\left(x_{j}\right)=\varphi\left(x_{j}^{+}\right) n_{I_{j}}\left(x_{j}\right)+\varphi\left(x_{j}^{-}\right) n_{I_{j+1}}\left(x_{j}\right)=-\varphi\left(x_{j}^{+}\right)+\varphi\left(x_{j}^{-}\right) .
$$

We also define

$$
\llbracket \varphi n \rrbracket(0)=-\varphi\left(0^{+}\right) \quad \text { and } \quad \llbracket \varphi n \rrbracket(1)=+\varphi\left(1^{-}\right) .
$$


TABLE 1. Numerical traces at the interior nodes.

\begin{tabular}{|c|c|c|}
\hline method & $\widehat{u}_{h}^{\epsilon}$ & $\widehat{q}_{h}$ \\
\hline $\begin{array}{l}\text { B.Z. [5] } \\
\text { Brezzi et al. [12] } \\
\text { B.O. [9] } \\
\text { NIPG [25] }\end{array}$ & $\begin{array}{c}\left\{u_{h}\right\}+n / 2 \llbracket u_{h} n \rrbracket \\
\left\{\left\{u_{h}\right\}+n / 2 \llbracket u_{h} n \rrbracket\right. \\
\left.\left\{u_{h}\right\}\right\}+n \llbracket u_{h} n \rrbracket \\
\left\{\left\{u_{h}\right\}+n \llbracket u_{h} n \rrbracket\right.\end{array}$ & $\begin{array}{c}-\alpha \llbracket u_{h} n \rrbracket \\
-\alpha_{r}\left(\llbracket u_{h} n \rrbracket\right) \\
\epsilon\left\{\left\{u_{h}^{\prime}\right\}\right. \\
\left.\epsilon\left\{u_{h}^{\prime}\right\}\right\}-\alpha \llbracket u_{h} n \rrbracket\end{array}$ \\
\hline $\begin{array}{c}\text { m-B.Z. } \\
\text { IP [7, 2] } \\
\text { Bassi et al. [8] } \\
\text { Brezzi et al. [11] } \\
\text { LDG [18] } \\
\text { DG [18, 14] } \\
\text { md-LDG }[18,15] \\
\text { md-DG }[18]\end{array}$ & $\begin{array}{c}\left\{\left\{u_{h}\right\}\right. \\
\left\{u_{h}\right\} \\
\left\{\left\{u_{h}\right\}\right. \\
\left\{u_{h}\right\} \\
\left\{u_{h}\right\}+\beta \llbracket u_{h} n \rrbracket \\
\left\{u_{h}\right\}+\beta \llbracket u_{h} n \rrbracket-\gamma \llbracket q_{h} n \rrbracket \\
\left.\left\{u_{h}\right\}\right]+\frac{1}{2} \llbracket u_{h} n \rrbracket=u_{h}^{-} \\
\left\{u_{h}\right\}+\frac{1}{2} \llbracket u_{h} n \rrbracket-\gamma \llbracket q_{h} n \rrbracket\end{array}$ & $\begin{array}{c}-\alpha \llbracket u_{h} n \rrbracket \\
\epsilon\left\{\left\{u_{h}^{\prime}\right\}-\alpha \llbracket u_{h} n \rrbracket\right. \\
\epsilon\left\{\left\{u_{h}^{\prime}\right\}-\alpha_{r}\left(\llbracket u_{h} n \rrbracket\right)\right. \\
\left\{q_{h}\right\}-\alpha_{r}\left(\llbracket u_{h} n \rrbracket\right) \\
\left\{\left\{q_{h}\right\}-\beta \llbracket q_{h} n \rrbracket-\alpha \llbracket u_{h} n \rrbracket\right. \\
\left\{\left\{q_{h}\right\}-\beta \llbracket q_{h} n \rrbracket-\alpha \llbracket u_{h} n \rrbracket\right. \\
\left\{\left\{q_{h}\right\}-\frac{1}{2} \llbracket q_{h} n \rrbracket=q_{h}^{+}\right. \\
\left\{\left\{q_{h}\right\}\right\}-\frac{1}{2} \llbracket q_{h} n \rrbracket\end{array}$ \\
\hline $\begin{array}{l}\text { h-R.T. }[24,3] \\
\text { DPG }[10]\end{array}$ & $\begin{array}{l}\text { new unknown } \\
\text { new unknown }\end{array}$ & $\begin{array}{c}q_{h} \\
\text { new unknown }\end{array}$ \\
\hline
\end{tabular}

TABLE 2. The numerical trace $\widehat{q}_{h}$ at the boundary.

\begin{tabular}{ccc}
\hline method & $\widehat{q}_{h}(0)$ & $\widehat{q}_{h}(1)$ \\
\hline \hline B.Z. [5] & $-\alpha\left(u_{D}(0)-u_{h}\left(0^{+}\right)\right)$ & $-\alpha\left(u_{h}\left(1^{-}\right)-u_{D}(1)\right)$ \\
Brezzi et al. [12] & $-\alpha_{r}\left(u_{D}(0)-u_{h}\left(0^{+}\right)\right)$ & $-\alpha_{r}\left(u_{h}\left(1^{-}\right)-u_{D}(1)\right)$ \\
B.O. [9] & $\epsilon u_{h}^{\prime}\left(0^{+}\right)$ & $\epsilon u_{h}^{\prime}\left(1^{-}\right)$ \\
NIPG [25] & $\epsilon u_{h}^{\prime}\left(0^{+}\right)-\alpha\left(u_{D}(0)-u_{h}\left(0^{+}\right)\right)$ & $\epsilon u_{h}^{\prime}\left(1^{-}\right)-\alpha\left(u_{h}\left(1^{-}\right)-u_{D}(1)\right)$ \\
\hline m-B.Z. & $-\alpha\left(u_{D}(0)-u_{h}\left(0^{+}\right)\right)$ & $-\alpha\left(u_{h}\left(1^{-}\right)-u_{D}(1)\right)$ \\
IP [7, 2] & $\epsilon u_{h}^{\prime}\left(0^{+}\right)-\alpha\left(u_{D}(0)-u_{h}\left(0^{+}\right)\right)$ & $\epsilon u_{h}^{\prime}\left(1^{-}\right)-\alpha\left(u_{h}\left(1^{-}\right)-u_{D}(1)\right)$ \\
Bassi et al. [8] & $\epsilon u_{h}^{\prime}\left(0^{+}\right)-\alpha\left(u_{D}(0)-u_{h}\left(0^{+}\right)\right)$ & $\epsilon u_{h}^{\prime}\left(1^{-}\right)-\alpha\left(u_{h}\left(1^{-}\right)-u_{D}(1)\right)$ \\
Brezzi et al. [11] & $q_{h}\left(0^{+}\right)-\alpha_{r}\left(u_{D}(0)-u_{h}\left(0^{+}\right)\right)$ & $q_{h}\left(1^{-}\right)-\alpha_{r}\left(u_{h}\left(1^{-}\right)-u_{D}(1)\right)$ \\
LDG [18] & $q_{h}\left(0^{+}\right)-\alpha\left(u_{D}(0)-u_{h}\left(0^{+}\right)\right)$ & $q_{h}\left(1^{-}\right)-\alpha\left(u_{h}\left(1^{-}\right)-u_{D}(1)\right)$ \\
DG [18, 14] & $q_{h}\left(0^{+}\right)-\alpha\left(u_{D}(0)-u_{h}\left(0^{+}\right)\right)$ & $q_{h}\left(1^{-}\right)-\alpha\left(u_{h}\left(1^{-}\right)-u_{D}(1)\right)$ \\
md-LDG [18, 15] & $q_{h}\left(0^{+}\right)$ & $q_{h}\left(1^{-}\right)-\alpha\left(u_{h}\left(1^{-}\right)-u_{D}(1)\right)$ \\
md-DG [18] & $q_{h}\left(0^{+}\right)$ & $q_{h}\left(1^{-}\right)-\alpha\left(u_{h}\left(1^{-}\right)-u_{D}(1)\right)$ \\
\hline h-R.T. [24, 3] & $q_{h}\left(0^{+}\right)$ & $q_{h}\left(1^{-}\right)$ \\
DPG [10] & new unknown & new unknown \\
\hline
\end{tabular}

TABle 3. Polynomial degree of the trial and test functions.

\begin{tabular}{ccccc}
\hline method & $\ell_{V_{h}}$ & $\ell_{W_{h}}$ & $\ell_{\mathbb{V}_{h}}$ & $\ell_{\mathbb{W}_{h}}$ \\
\hline h-R.T. [24,3] & $p+1$ & $p$ & $p+1$ & $p$ \\
DPG [10] & $p$ & $p$ & $p+1$ & $p+1$ \\
\hline
\end{tabular}

The numerical traces $\widehat{q}_{h}$ at the boundary are displayed in Table 2 . In there, the penalization parameter at the border is typically

$$
\alpha(0)=\alpha(1)=\epsilon p / h .
$$

The numerical trace associated with the convection is the classical upwinding trace, namely,

$$
\widehat{u}_{h}^{c}(0)=u_{D}(0) \quad \text { and } \quad \widehat{u}_{h}^{c}\left(x_{i}\right)=u_{h}\left(x_{i}^{-}\right)
$$

for all the remaining nodes. 
Note that to define the method of Bassi et al. [8], and the methods of Brezzi et al. $[11,12]$ we need to define the lifting operator $\alpha_{r}$ as follows. Given $x_{j} \in \mathscr{E}_{h}$ we define the lifting $r_{x_{j}}(\eta) \in \mathbb{V}_{h}$ of $\eta$ by the condition

$$
\left(r_{x_{j}}(\eta), \tau\right)_{\Omega_{h}}:=-\eta\left(x_{j}\right)\left\{\left\{\tau\left(x_{j}\right)\right\} \quad \text { for all } \tau \in \mathbb{V}_{h} .\right.
$$

We then set $\left[\alpha_{r}(\eta)\right]\left(x_{j}\right):=\mu_{x_{j}}\left\{\left\{r_{x_{j}}(\eta)\right\}\left(x_{j}\right)\right.$. Here $\mu_{x_{j}}$ is a positive parameter. Note that $r_{x_{j}}(\eta)$ vanishes outside one or two elements containing $x_{j}$ and that $r(\eta)=$ $\sum_{j=0}^{N} r_{x_{j}}(\eta)$.

Finally, let us describe the spaces of these methods. Since all the spaces are of the form

$$
X_{h}^{\ell}=\left\{w \in L^{2}\left(\Omega_{h}\right):\left.w\right|_{I_{j}} \in P^{\ell}\left(I_{j}\right), j=1, \ldots, N\right\},
$$

where $\ell$ is a natural number, they are characterized by the single natural number $\ell$. For all the numerical methods of the first two groups, we take all the spaces equal to $X_{h}^{p}$, that is,

$$
\ell_{V_{h}}=\ell_{W_{h}}=\ell_{\mathbb{V}_{h}}=\ell_{\mathbb{W}_{h}}=p .
$$

In Table 3 we display such a number for the methods of the third group.

2.3. Superconvergence at the nodes. Our main result identifies quantities u and flx that always superconverge to the values of the potential $u$ and the flux $(-q+c u)$, respectively, at each of the nodes. Let us define them.

If $x_{i}$ is a boundary node, we have

$$
\mathrm{u}\left(x_{i}\right):=u_{D}\left(x_{i}\right),
$$

and if $x_{i}$ is an interior node,

$$
\mathrm{u}\left(x_{i}\right):=\left\{\left\{\widehat{u}_{h}^{\epsilon}\right\}\left(x_{i}\right)+\left\langle 1, \epsilon\left\{\left\{\varphi_{x_{i}}^{\prime}\right\} \llbracket \llbracket \widehat{u}_{h}^{\epsilon} n \rrbracket+\varphi_{x_{i}} \llbracket\left(-\widehat{q}_{h}+c \widehat{u}_{h}^{c}\right) n \rrbracket\right\rangle_{\mathscr{E}_{h}} .\right.\right.
$$

Here, for any given point $y \in(0,1), \varphi_{y}$ is the solution of

$$
\begin{array}{ll}
-\left(\epsilon \varphi_{y}^{\prime \prime}+c \varphi_{y}^{\prime}\right)=0 & \text { in } \Omega \backslash\{y\}, \\
\llbracket \varphi_{y} n \rrbracket(y)=0, & \epsilon \llbracket \varphi_{y}^{\prime} n \rrbracket(y)=1, \\
\varphi_{y}=0 & \text { in } \partial \Omega,
\end{array}
$$

$\mathscr{E}_{h}$ denotes the set of interior nodes, and

$$
\langle\zeta, \xi\rangle_{\mathscr{E}_{h}^{\circ}}:=\sum_{i=1}^{N-1} \zeta\left(x_{i}\right) \xi\left(x_{i}\right),
$$

for any functions $\zeta$ and $\xi$ defined on $\mathscr{E}_{h}$.

Similarly, if $x_{i}$ is a boundary node,

$$
\mathrm{flx}\left(x_{i}\right):=\left(-\widehat{q}_{h}+c \widehat{u}_{h}^{c}\right)\left(x_{i}\right)+\left\langle 1, \epsilon \psi_{x_{i}}^{\prime} \llbracket \widehat{u}_{h}^{\epsilon} n \rrbracket+\left\{\left\{\psi_{x_{i}}\right\} \llbracket\left(-\widehat{q}_{h}+c \widehat{u}_{h}^{c}\right) n \rrbracket\right\rangle_{\mathscr{E}_{h}^{\circ}}\right.
$$

and if $x_{i}$ is an interior node,

$$
\mathrm{flx}\left(x_{i}\right):=\left\{\left\{-\widehat{q}_{h}+c \widehat{u}_{h}^{c}\right\}\right\}\left(x_{i}\right)+\left\langle 1, \epsilon \psi_{x_{i}}^{\prime} \llbracket \widehat{u}_{h}^{\epsilon} n \rrbracket+\left\{\left\{\psi_{x_{i}}\right\} \llbracket \llbracket\left(-\widehat{q}_{h}+c \widehat{u}_{h}^{c}\right) n \rrbracket\right\rangle_{\mathscr{E}_{h}^{\circ}} .\right.
$$


Here, for any point $y \in(0,1), \psi_{y}$ is the solution of

$$
\begin{aligned}
& -\left(\epsilon \psi_{y}^{\prime \prime}+c \psi_{y}^{\prime}\right)=0 \quad \text { in } \Omega \backslash\{y\}, \\
& \llbracket \psi_{y} n \rrbracket(y)=1, \quad \epsilon \llbracket \psi_{y}^{\prime} n \rrbracket(y)=0, \\
& \psi_{y}=0 \quad \text { in } \partial \Omega \text {. }
\end{aligned}
$$

We also define, for $z \in\{0,1\}$,

$$
\left(\varphi_{z}, \psi_{z}\right):=\lim _{y \rightarrow z}\left(\varphi_{y}, \psi_{y}\right) .
$$

Note that this implies that the function $\left(\varphi_{z}, \psi_{z}\right)$ is identically zero for $z \in\{0,1\}$.

The estimate of the errors

$$
\mathrm{e}_{\mathrm{u}}:=u-\mathrm{u} \quad \text { and } \quad \mathrm{e}_{\mathrm{flx}}:=(-q+c u)-\mathrm{flx}
$$

is going to be given in terms of the $L^{2}\left(\Omega_{h}\right)$-norm of the errors

$$
e_{u}:=u-u_{h} \quad \text { and } \quad e_{q}:=q-q_{h},
$$

and $H^{s}\left(\Omega_{h}\right)$-seminorms of the Green's functions $\varphi_{x_{i}}$ and $\psi_{x_{i}}$. The $H^{s}\left(\Omega_{h}\right)$-seminorm of a function $w$ is given by $|w|_{s, \Omega_{h}}:=\left(\sum_{j=1}^{N}|w|_{s, I_{j}}^{2}\right)^{\frac{1}{2}}$, where $|w|_{s, I_{j}}:=$ $\left\|w^{(s)}\right\|_{0, I_{j}}$ and $\|\cdot\|_{0, I_{j}}$ is the $L^{2}$-norm on $I_{j}$.

We are now ready to state our main result.

Theorem 2.1. Consider any well-defined method whose approximate solution satisfies the weak formulation (2.2). Suppose that the spaces of test functions $\mathbb{V}_{h}$ and $\mathbb{W}_{h}$ contain the space $X_{h}^{\ell}$ where $\ell \geq 1$. Then,

$$
\begin{aligned}
& \left|\mathrm{e}_{\mathrm{u}}\left(x_{i}\right)\right| \leq C_{s}\left\|\left(e_{u}, e_{q}\right)\right\|_{c, \Omega_{h}}\left|\varphi_{x_{i}}\right|_{s+1, \Omega_{h}}\left(\frac{h^{\min (s, \ell)}}{\ell^{s}}\right), \\
& \left|\mathrm{e}_{\mathrm{fl} \mid}\left(x_{i}\right)\right| \leq C_{s}\left\|\left(e_{u}, e_{q}\right)\right\| \|_{c, \Omega_{h}}\left|\psi_{x_{i}}\right|_{s+1, \Omega_{h}}\left(\frac{h^{\min (s, \ell)}}{\ell^{s}}\right),
\end{aligned}
$$

for all nodes $x_{i}$. Here,

$$
\left\|\left(e_{u}, e_{q}\right)\right\|_{c, \Omega_{h}}:=\left\|e_{q}\right\|_{0, \Omega_{h}}+c\left\|e_{u}\right\|_{0, \Omega_{h}},
$$

and the constant $C_{s}$ only depends on $s \geq 0$.

Note that, given $\epsilon, c$ and $s$, the quantities $\left|\varphi_{x_{i}}\right|_{s+1, \Omega_{h}}$ and $\left|\psi_{x_{i}}\right|_{s+1, \Omega_{h}}$ can be bounded independently of $i$ and $h$.

Next, we discuss several important consequences of this result.

- Methods with conservative numerical traces. When the numerical traces are conservative, Theorem 2.1 is a superconvergence result for them, thanks to the following simple result.

Proposition 2.2. Assume that the numerical traces $\widehat{u}_{h}^{\epsilon}$ and are $\left(-\widehat{q}_{h}+c \widehat{u}_{h}^{c}\right)$ are conservative. Then

$$
\mathbf{u}=\widehat{u}_{h}^{\epsilon} \quad \text { and } \quad \mathrm{flx}=\left(-\widehat{q}_{h}+c \widehat{u}_{h}^{c}\right),
$$

on all the nodes of the mesh. 
TABLE 4. Order of convergence $p^{\star}$ of both numerical traces of conservative methods guaranteed by Theorem 2.1.

\begin{tabular}{cccccc}
\hline method & $\left.\alpha\right|_{\mathscr{E}_{h}^{\circ}}$ & $\left.\beta\right|_{\mathscr{E}_{h}^{\circ}}$ & $\left.\gamma\right|_{\mathscr{E}_{h}^{\circ}}$ & $\left\|\left(e_{u}, e_{q}\right)\right\|_{c, \Omega_{h}}$ & $p^{\star}$ \\
\hline IP [7, 2] & $\epsilon p / h$ & - & - & $p$ & $2 p$ \\
Bassi et al. [8] & - & - & - & $p$ & $2 p$ \\
Brezzi et al. [11] & - & - & - & $p$ & $2 p$ \\
LDG [18] & $\epsilon p / h$ & 0 & 0 & $p$ & $2 p$ \\
LDG [18] & $\epsilon p / h$ & $1 / 2$ & 0 & $p$ & $2 p$ \\
DG [18, 14] & $\epsilon p / h$ & 0 & $h / p$ & $p$ & $2 p$ \\
md-LDG [18] & 0 & $1 / 2$ & 0 & $p+1$ & $2 p+1$ \\
md-DG [18, 14] & 0 & $1 / 2$ & $h / p$ & $p+1$ & $2 p+1$ \\
\hline h-R.T. [24, 3] & - & - & - & $p+1$ & $2 p+1$ \\
DPG [10] & - & - & - & $p+1$ & $2 p+2$ \\
& & & & & \\
\hline
\end{tabular}

Proof. Conservativity of the numerical traces $\widehat{u}_{h}^{\epsilon}$ and $\left(-\widehat{q}_{h}+c \widehat{u}_{h}^{c}\right)$ means that (see [4]) for all interior nodes $x_{i}$,

$$
\llbracket \widehat{u}_{h}^{\epsilon} n \rrbracket\left(x_{i}\right)=0 \quad \text { and } \quad \llbracket\left(-\widehat{q}_{h}+c \widehat{u}_{h}^{c}\right) n \rrbracket\left(x_{i}\right)=0 .
$$

By the definitions of $u$ and $f \mathbf{x},(2.3)$ and (2.5), respectively, this immediately implies the result.

A straightforward application of Theorem 2.1 is summarized in Table 4, where we display the orders of superconvergence of the numerical traces of several methods. To obtain them, all we need to do is to provide estimates for $\left\|e_{u}\right\|_{0, \Omega_{h}}$ and $\left\|e_{q}\right\|_{0, \Omega_{h}}$. For the purely elliptic case, these estimates are available in [4] for the first six DG methods, in [24] for the h-R.T. method, and in [10] for the DPG method. Similar estimates for the convection-diffusion case can be easily obtained.

For the md-LDG and md-DG methods, such estimates can be obtained by a suitable modification of the analysis proposed in [15] for the time-dependent convectiondiffusion problem. On the other hand, Theorem 2.6, which is a variation on our main result that takes into account the special structure of these methods, also implies a superconvergence of order $2 p+1$ of the numerical traces of the md-LDG method and the md-DG method with $\gamma=h / p$.

All the orders of convergence in Table 4 have been numerically verified to be sharp.

- Postprocessing. When the numerical method has conservative numerical traces, it is possible to use their superconvergence to construct a new approximate solution $\left(q_{h}^{\star}, u_{h}^{\star}\right)$ that converges uniformly to the exact solution with the same order of convergence of the numerical traces. This can be achieved by a straightforward extension of the procedure used in [17] in the framework of DG methods for Timoshenko beams.

Next we define this postprocessing. On the element $I_{j}, 1 \leq j \leq N$, we define $\left(q_{h}^{*}, u_{h}^{*}\right)$ as the element of the space $\left[P^{p^{*}}\left(I_{j}\right)\right]$ for some $p<p^{*} \leq 2 p$ such that

$$
\begin{aligned}
\left(q_{h}^{*}, w^{\prime}\right)_{I_{j}} & +\left(\frac{c}{\epsilon} q_{h}^{*}, w\right)_{I_{j}}-q_{h}^{*}\left(x_{j}^{-}\right) w\left(x_{j}^{-}\right) \\
& =(f, w)_{I_{j}}-\left(\widehat{q}_{h}-c \widehat{u}_{h}^{c}+c \widehat{u}_{h}^{\epsilon}\right)\left(x_{j-1}\right) w\left(x_{j-1}^{+}\right), \\
-\left(\epsilon u_{h}^{*}, v^{\prime}\right)_{I_{j}} & +\epsilon u_{h}^{*}\left(x_{j}^{-}\right) v\left(x_{j}^{-}\right)=\left(q_{h}^{*}, v\right)_{I_{j}}+\epsilon \widehat{u}_{h}^{\epsilon}\left(x_{j-1}\right) v\left(x_{j-1}^{+}\right)
\end{aligned}
$$

for all $v, w \in P^{p^{*}}\left(I_{j}\right)$. 
It is not difficult to see that the above equations are the discretization by the classical DG method of the following simple set of initial value problems for ODEs:

$$
\begin{aligned}
& -\left(q^{*}\right)^{\prime}+\frac{c}{\epsilon} q^{*}=f \quad \text { in } I_{j}, \quad q^{*}\left(x_{j-1}\right)=\left(\widehat{q}_{h}-c \widehat{u}_{h}^{c}+c \widehat{u}_{h}^{\epsilon}\right)\left(x_{j-1}\right), \\
& \epsilon\left(u^{*}\right)^{\prime}=q^{*} \quad \text { in } I_{j}, \quad u^{*}\left(x_{j-1}\right)=\widehat{u}_{h}^{\epsilon}\left(x_{j-1}\right) .
\end{aligned}
$$

Both this system of ODEs as well as its discrete counterpart are extremely simple to solve. Indeed, we first obtain $q_{h}^{*}$ by solving $(2.9 \mathrm{a})$, we then insert it into $(2.9 \mathrm{~b})$ to solve for $u_{h}^{*}$. Note that to compute this postprocessing, all we need is to invert only two matrices of order $\left(p^{*}+1\right)$ which are the same for all the elements. The first is the stiffness matrix associated with the equations (2.9a), and the second the matrix associated with $(2.9 \mathrm{~b})$. Thus the overall computational cost of this postprocessing is negligible as compared to the cost of actually computing the approximate solution.

As an application of our superconvergence results at the nodes we provide the main approximation property of the postprocessed solution. It is given in terms of the norm given by $\|u\|_{\infty, \Omega_{h}}:=\max _{1 \leq j \leq N}\left(\sup _{x \in I_{j}}|u(x)|\right)$.

Proposition 2.3. Let $\left(q_{h}, u_{h}\right)$ be the approximate solution by any of the methods under consideration with conservative numerical traces. Suppose further that

$$
\left|\left(u-\widehat{u}_{h}^{\epsilon}\right)\left(x_{i}\right)\right|+\left|(q-c u)\left(x_{i}\right)-\left(\widehat{q}_{h}-c \widehat{u}_{h}^{c}\right)\left(x_{i}\right)\right| \leq C h^{k}
$$

for all $x_{i} \in \mathscr{E}_{h}$. Then, the error of the postprocessed approximation is such that

$$
\left\|q-q_{h}^{*}\right\|_{\infty, \Omega_{h}}+\left\|u-u_{h}^{*}\right\|_{\infty, \Omega_{h}} \leq D h^{\min \left(p^{*}+1, k\right)}
$$

for some constant $D$ independent of $h$.

The proof of this result is a variation of a similar result proven in [17]. Note that for most of the conservative methods in Table 1 the superconvergence is of order $2 p$, hence one can take $p^{*}=2 p$. As a result, $\left(q_{h}^{*}, u_{h}^{*}\right)$ will converge to the exact solution with order $2 p$, uniformly in the whole domain. For some particular methods, such as md-LDG or h-R.T., the convergence of the postprocessed solution is of order $2 p+1$.

- Exactness of the numerical traces for the purely elliptic case $c=0$.

It is very well known that in the purely elliptic case, the nodal values of the conforming finite element method coincide with the exact solution. A similar result holds for LDG methods: the numerical traces for the potential are also exact for LDG methods; see [13]. In [22], the numerical trace for the flux of the IP method was shown to be exact. These are particular cases of the following result.

Corollary 2.4. Assume that the hypotheses of Theorem 2.1 are satisfied. Assume also that the numerical traces are conservative. Then, for the purely elliptic case, $c=0$, we have that, for all nodes,

$$
\widehat{u}_{h}^{\epsilon}=u \quad \text { and } \quad \widehat{q}_{h}=q .
$$

In other words, for the methods with conservative numerical traces, both numerical traces are exact regardless of the way they are actually defined. In particular, this holds the m-B.Z. method even though its numerical trace for the flux is not consistent. It also holds for any well-defined DG method, independently of the actual values of the parameters $\alpha, \gamma$, and $\beta$ defining their numerical traces. 
For example, it was shown in [16] that the LDG method with $\beta=0$ is well defined not only for $\alpha>0$, but also for

$$
\alpha<-\frac{3(2 p+1)}{2 h},
$$

for uniform meshes. Thus, the numerical fluxes are exact for all these values of $\alpha$. This indicates that the so-called penalization parameter $\alpha$ can be negative without having a detrimental impact on the numerical traces.

This raises the issue of what is the effect of the actual definition of the numerical traces on the approximate solution $\left(q_{h}, u_{h}\right)$. The following result identifies the degrees of freedom that are completely independent of the actual definition of the numerical traces. We denote by $\mathbb{P}_{h}^{\ell}$ the $L^{2}$-projection into the space $X_{h}^{\ell}$.

Corollary 2.5. Assume that the hypotheses of Theorem 2.1 are satisfied. Assume also that the numerical traces are conservative. Then, for the purely elliptic case, $c=0$, we have that,

$$
\mathbb{P}_{h}^{\ell_{\mathbb{W}_{h}}-1} q_{h}=\mathbb{P}_{h}^{\ell_{\mathbb{W}_{h}}-1} q \quad \text { and } \quad \mathbb{P}_{h}^{\min \left\{\ell_{\mathbb{V}_{h}}-1, \ell_{\mathbb{W}_{h}}-2\right\}} u_{h}=\mathbb{P}_{h}^{\min \left\{\ell_{\mathbb{V}_{h}}-1, \ell_{\mathbb{W}_{h}}-2\right\}} u .
$$

This implies that only the degrees of freedom associated to the Legendre polynomials of highest degree are affected by the definition of the numerical traces. Take, for example, the DG methods under consideration. For those methods, we have $p=\ell_{\mathbb{V}_{h}}=\ell_{\mathbb{W}_{h}}$, and so

$$
\mathbb{P}_{h}^{(p-1)} q_{h}=\mathbb{P}_{h}^{(p-1)} q \quad \text { and } \quad \mathbb{P}_{h}^{(p-2)} u_{h}=\mathbb{P}_{h}^{(p-2)} u .
$$

Thus, if we write, for $x$ in the element $I_{j}$,

$$
q_{h}(x)=\sum_{k=0}^{p} q_{k}^{j} L_{k}\left(T_{j}(x)\right) \quad \text { and } \quad u_{h}(x)=\sum_{k=0}^{p} u_{k}^{j} L_{k}\left(T_{j}(x)\right),
$$

where $T_{j}(x)=\left(2 x-x_{j}-x_{j-1}\right) /\left(x_{j}-x_{j-1}\right)$ and $L_{k}(\cdot)$ is the Legendre polynomial of degree $k$, the above result states that only $q_{p}^{j}, u_{p-1}^{j}$ and $u_{p}^{j}$ are actually affected by the definition of the numerical traces.

Let us end by pointing out that we can use the last two corollaries to construct a new approximation that is not affected by the definition of the numerical traces. Let us illustrate this with the DG methods under consideration. On each interval $I_{j}$, we define the function $u_{h}^{\star}$ to be the polynomial of degree $p+2$ such that

$$
\begin{array}{ll}
u_{h}^{\star}\left(x_{i}\right)=\widehat{u}_{h}^{\epsilon}\left(x_{i}\right), & i \in\{j-1, j\}, \\
\left(u_{h}^{\star}\right)^{\prime}\left(x_{i}\right)=\frac{1}{\epsilon} \widehat{q}_{h}\left(x_{i}\right), & i \in\{j-1, j\}, \\
\mathbb{P}_{h}^{(p-2)}\left(u_{h}^{\star}\right)=\mathbb{P}_{h}^{(p-2)} u_{h} . &
\end{array}
$$

Then we immediately have that $u-u_{h}^{\star}$ and $h\left(u-u_{h}^{\star}\right)^{\prime}$ are of order $h^{p+3}$ pointwise in the whole domain.

- Methods with nonconservative numerical traces. The B.Z., B.O. and the NIPG methods do not appear in Table 4 because although their numerical trace $\left(-\widehat{q}_{h}+c \widehat{u}_{h}^{c}\right)$ is conservative, their numerical trace $\widehat{u}_{h}^{\epsilon}$ is not. On the other hand, Theorem 2.1 still gives useful information for these schemes. Indeed, since in this 
case we have

$$
\begin{aligned}
& \mathrm{u}\left(x_{i}\right)=\left\{\left\{\widehat{u}_{h}^{\epsilon}\right\}\left(x_{i}\right)+\mathrm{m}\left\langle\left\{\left\{\epsilon \varphi_{x_{i}}^{\prime}\right\}, \llbracket u_{h} n \rrbracket\right\rangle_{\mathscr{E}_{h}^{\circ}},\right.\right. \\
& \mathrm{flx}\left(x_{i}\right)=\left(\widehat{q}_{h}+c \widehat{u}_{h}^{c}\right)\left(x_{i}\right)+\mathrm{m}\left\langle\epsilon \psi_{x_{i}}^{\prime}, \llbracket u_{h} n \rrbracket\right\rangle_{\mathscr{E}_{h}^{\circ},}
\end{aligned}
$$

with $\mathrm{m}=1$ for B.Z. and $\mathrm{m}=2$ for B.O. and NIPG, and since

$$
\left\{\left\{\widehat{u}_{h}^{\epsilon}\right\}\left(x_{i}\right)=\left\{\left\{u_{h}\right\}\right\}\left(x_{i}\right)\right. \text {, }
$$

we readily obtain that

$$
\begin{array}{r}
\mid\left(u-\left\{\left\{u_{h}\right\}\right)\left(x_{i}\right)|\leq| \mathrm{e}_{\mathrm{u}}\left(x_{i}\right) \mid+C \epsilon\left\|\varphi_{x_{i}}^{\prime}\right\|_{1, \Omega_{h}}\left(\sum_{i=1}^{N-1} \frac{1}{h} \llbracket u_{h} n \rrbracket^{2}\right)^{\frac{1}{2}},\right. \\
\left|\left((-q+c u)-\left(-\widehat{q}_{h}+c \widehat{u}_{h}^{c}\right)\right)\left(x_{i}\right)\right| \leq\left|\mathrm{e}_{\mathrm{flx}}\left(x_{i}\right)\right|+C \epsilon\left\|\psi_{x_{i}}^{\prime}\right\|_{1, \Omega_{h}}\left(\sum_{i=1}^{N-1} \frac{1}{h} \llbracket u_{h} n \rrbracket^{2}\right)^{\frac{1}{2}} .
\end{array}
$$

Thus, the order of convergence of $\left\{\left\{u_{h}\right\}\right\}$ and $\left(-\widehat{q}_{h}+c \widehat{u}_{h}^{c}\right)$ is given by the order of convergence of the global quantity $\left(\sum_{i=1}^{N-1} h^{-1} \llbracket u_{h} n \rrbracket^{2}\right)^{1 / 2}$. Larson and Niklasson [22] recently proved that for the NIPG method this quantity converges with order $p$ for the purely elliptic case.

2.4. Another version of the main result for the md-LDG and the mdDG methods. Here we consider a variation of the main result for two special DG methods, namely, the md-LDG and the md-DG method. The result does not require estimates for $\left\|e_{u}\right\|_{0, \Omega_{h}}$ and $\left\|e_{q}\right\|_{0, \Omega_{h}}$. Instead, it requires an estimate for the quantity

$$
\left|\left(\pi^{+} e_{q}, \pi^{-} e_{u}\right)\right|_{\mathcal{A}_{h}},
$$

where the seminorm $|(\cdot, \cdot)|_{\mathcal{A}_{h}}$ is given by

$$
\left|\left(q_{h}, u_{h}\right)\right|_{\mathcal{A}_{h}}:=\left(\frac{1}{\epsilon}\left\|q_{h}\right\|_{0, \Omega_{h}}^{2}+\frac{1}{2}\left\langle c, \llbracket u_{h} n \rrbracket^{2}\right\rangle_{\mathscr{E}_{h}}+\left\langle\gamma, \llbracket q_{h} n \rrbracket^{2}\right\rangle_{\mathscr{E}_{h}^{\circ}}+\alpha u_{h}^{2}\left(1^{-}\right)\right)^{1 / 2},
$$

and the operators $\pi^{+}$and $\pi^{-}$are defined as follows. If $\phi \in H^{1}\left(\Omega_{h}\right)$, then $\pi^{ \pm} \phi$ is the function $X_{h}^{p}$ such that, for each element $I_{j}=\left(x_{j-1}, x_{j}\right)$ of the mesh $\Omega_{h}$,

$$
\begin{aligned}
& \left(\pi^{ \pm} \phi-\phi, v\right)_{I_{j}}=0 \quad \forall v \in P^{p-1}\left(I_{j}\right), \text { if } p>0, \\
& \left(\pi^{-} \phi\right)\left(x_{j}^{-}\right)=\phi\left(x_{j}^{-}\right), \quad\left(\pi^{+} \phi\right)\left(x_{j-1}^{+}\right)=\phi\left(x_{j-1}^{+}\right) .
\end{aligned}
$$

Theorem 2.6. For the $m d-L D G$ and the $m d-D G$ methods, we have

$$
\begin{array}{r}
\left|{\widehat{e_{u}}}^{\epsilon}\left(x_{i}\right)\right| \leq C_{s}\left\|\left(e_{q}, e_{u}\right)\right\| \|_{\mathcal{A}_{h}, \Omega_{h}}\left|\varphi_{x_{i}}\right|_{s+1, \Omega_{h}}\left(\frac{h^{\min (s, p)}}{p^{s}}\right), \\
\left|\widehat{e_{q}}-c{\widehat{e_{u}}}^{c}\left(x_{i}\right)\right| \leq C_{s}\left\|\left(e_{q}, e_{u}\right)\right\|_{\mathcal{A}_{h}, \Omega_{h}}\left|\psi_{x_{i}}\right|_{s+1, \Omega_{h}}\left(\frac{h^{\min (s, p)}}{p^{s}}\right),
\end{array}
$$

for all the nodes $x_{i}$ and for all $s \geq 0$. Here

$$
\left\|\left(e_{q}, e_{u}\right)\right\|_{\mathcal{A}_{h}, \Omega_{h}}:=\left\|q-\pi^{+} q\right\|_{0, \Omega_{h}}+\sqrt{\epsilon}\left|\left(\pi^{+} e_{q}, \pi^{-} e_{u}\right)\right|_{\mathcal{A}_{h}},
$$

and the constant $C_{s}$ depends only on $s$. 
Since, by Proposition 5.1,

$$
\left\|\left(e_{q}, e_{u}\right)\right\|_{\mathcal{A}_{h}, \Omega_{h}} \leq 2\left(\left\|\pi^{+} q-q\right\|_{0, \Omega_{h}}^{2}+\left\langle\epsilon \gamma, \llbracket \pi^{+} q n \rrbracket^{2}\right\rangle_{\mathscr{E}_{h}}+\frac{\epsilon}{\alpha}\left|\left(\pi^{+} q-q\right)(1)\right|^{2}\right)^{1 / 2},
$$

for $\gamma$ of order $h / p \epsilon$ we get, by the approximation properties of the operators $\pi^{ \pm}$,

$$
\left\|\left(e_{q}, e_{u}\right)\right\| \|_{\mathcal{A}_{h}, \Omega_{h}} \leq C_{\tau}|q|_{\tau+1, \Omega_{h}}\left(\frac{h^{\min (\tau, p)+1}}{p^{\tau+1}}\right),
$$

where $C_{\tau}$ only depends on $\tau$. This immediately implies that

$$
\begin{array}{r}
\left|{\widehat{e_{u}}}^{\epsilon}\left(x_{i}\right)\right| \leq C_{s} C_{\tau}|q|_{\tau+1, \Omega_{h}}\left|\varphi_{x_{i}}\right|_{s+1, \Omega_{h}}\left(\frac{h^{\min (s, p)+\min (\tau, p)+1}}{p^{s+\tau+1}}\right), \\
\left|\widehat{e_{q}}-c{\widehat{e_{u}}}^{c}\left(x_{i}\right)\right| \leq C_{s} C_{\tau}|q|_{\tau+1, \Omega_{h}}\left|\psi_{x_{i}}\right|_{s+1, \Omega_{h}}\left(\frac{h^{\min (s, p)+\min (\tau, p)+1}}{p^{s+\tau+1}}\right) .
\end{array}
$$

Hence, the rate of convergence of the numerical traces of the md-methods is of order $(2 p+1) /\left(p^{2 p+1}\right)$ for sufficiently smooth exact solutions.

\section{Proofs}

3.1. The error representation formulas. The proofs of our main results are based on the following key result. To state it, we need to introduce some notation. Let $\mathscr{E}_{h}$ denote the set of nodes, and

$$
\langle\zeta, \xi\rangle_{\mathscr{E}_{h}}:=\sum_{i=0}^{N} \zeta\left(x_{i}\right) \xi\left(x_{i}\right),
$$

for any functions $\zeta$ and $\xi$ defined on $\mathscr{E}_{h}$.

Lemma 3.1 (Error representation). Let $x_{i}$ be any node in $\mathscr{E}_{h}$ and let $\left(Z_{h}, \zeta_{h}\right)$ be any function in the space $\mathbb{V}_{h} \times \mathbb{W}_{h}$. Then

$$
\begin{aligned}
e_{\mathrm{u}}\left(x_{i}\right)= & \left\langle 1, \llbracket \epsilon{\widehat{e_{u}}}^{\epsilon}\left(\varphi_{x_{i}}^{\prime}-Z_{h}\right) n \rrbracket\right\rangle_{\mathscr{E}_{h}^{\circ}}-\left\langle 1, \llbracket\left({\widehat{e_{q}}}-c{\widehat{e_{u}}}^{c}\right)\left(\varphi_{x_{i}}-\zeta_{h}\right) n \rrbracket\right\rangle_{\mathscr{E}_{h}} \\
& +\left(e_{q},\left(Z_{h}-\varphi_{x_{i}}^{\prime}\right)-\left(\zeta_{h}-\varphi_{x_{i}}\right)^{\prime}\right)_{\Omega_{h}}+\left(e_{u}, \epsilon\left(Z_{h}-\varphi_{x_{i}}^{\prime}\right)^{\prime}+c\left(\zeta_{h}-\varphi_{x_{i}}\right)^{\prime}\right)_{\Omega_{h}}
\end{aligned}
$$

and

$$
\begin{aligned}
e_{\mathrm{flx}}\left(x_{i}\right)= & \left\langle 1, \llbracket \epsilon{\widehat{e_{u}}}^{\epsilon}\left(\psi_{x_{i}}^{\prime}-Z_{h}\right) n \rrbracket\right\rangle_{\mathscr{E}_{h}^{\circ}}-\left\langle 1, \llbracket\left({\widehat{e_{q}}}^{\prime}-c{\widehat{e_{u}}}^{c}\right)\left(\psi_{x_{i}}-\zeta_{h}\right) n \rrbracket\right\rangle_{\mathscr{E}_{h}} \\
& +\left(e_{q},\left(Z_{h}-\psi_{x_{i}}^{\prime}\right)-\left(\zeta_{h}-\psi_{x_{i}}\right)^{\prime}\right)_{\Omega_{h}}+\left(e_{u}, \epsilon\left(Z_{h}-\psi_{x_{i}}^{\prime}\right)^{\prime}+c\left(\zeta_{h}-\psi_{x_{i}}\right)^{\prime}\right)_{\Omega_{h}} .
\end{aligned}
$$

To prove this result, we need the following identity.

Lemma 3.2 (Basic identity). Let $(Z, \zeta)$ be a solution of

$$
Z=\zeta^{\prime} \quad \text { and } \quad-\left(\epsilon Z^{\prime}+c \zeta^{\prime}\right)=0 \quad \text { in } \Omega_{h} .
$$

Set

$$
\Xi_{i}:=\left\langle 1, \llbracket \epsilon{\widehat{e_{u}}}^{\epsilon} Z n \rrbracket\right\rangle_{\mathscr{E}_{h}}-\left\langle 1, \llbracket\left({\widehat{e_{q}}}^{-c{\widehat{e_{u}}}^{c}}\right) \zeta n \rrbracket\right\rangle_{\mathscr{E}_{h}}
$$

Then

$$
\begin{aligned}
\Xi_{i}= & \left\langle 1, \llbracket \epsilon{\widehat{e_{u}}}^{\epsilon}\left(Z-Z_{h}\right) n \rrbracket\right\rangle_{\mathscr{E}_{h}}-\left\langle 1, \llbracket\left({\widehat{e_{q}}}-c{\widehat{e_{u}}}^{c}\right)\left(\zeta-\zeta_{h}\right) n \rrbracket\right\rangle_{\mathscr{E}_{h}} \\
& +\left(e_{q},\left(Z_{h}-Z\right)-\left(\zeta_{h}-\zeta\right)^{\prime}\right)_{\Omega_{h}}+\left(e_{u}, \epsilon\left(Z_{h}-Z\right)^{\prime}+c\left(\zeta_{h}-\zeta\right)^{\prime}\right)_{\Omega_{h}},
\end{aligned}
$$

for all $\left(Z_{h}, \zeta_{h}\right) \in \mathbb{V}_{h} \times \mathbb{W}_{h}$. 
Proof. Since

$$
\begin{aligned}
\Xi_{i}= & \left\langle 1, \llbracket \epsilon{\widehat{e_{u}}}^{\epsilon}\left(Z-Z_{h}\right) n \rrbracket\right\rangle_{\mathscr{E}_{h}}-\left\langle 1, \llbracket\left({\widehat{e_{q}}}^{-c{\widehat{e_{u}}}^{c}}\right)\left(\zeta-\zeta_{h}\right) n \rrbracket\right\rangle_{\mathscr{E}_{h}} \\
& +\left\langle 1, \llbracket \epsilon{\widehat{e_{u}}}^{\epsilon} Z n \rrbracket\right\rangle_{\mathscr{E}_{h}}-\left\langle 1, \llbracket\left(\widehat{e_{q}}-c{\widehat{e_{u}}}^{c}\right) \zeta n \rrbracket\right\rangle_{\mathscr{E}_{h}},
\end{aligned}
$$

we only have to prove that the last term of the above identity is equal to

$$
\left(e_{q},\left(Z_{h}-Z\right)-\left(\zeta_{h}-\zeta\right)^{\prime}\right)_{\Omega_{h}}+\left(e_{u}, \epsilon\left(Z_{h}-Z\right)^{\prime}+c\left(\zeta_{h}-\zeta\right)^{\prime}\right)_{\Omega_{h}} .
$$

To do that, we proceed as follows. Subtracting equations (2.2) from equations (2.1), we get

$$
\begin{aligned}
\left\langle\epsilon{\widehat{e_{u}}}^{\epsilon}, v n\right\rangle_{\partial \Omega_{h}} & =\left(e_{q}, v\right)_{\Omega_{h}}+\left(\epsilon e_{u}, v^{\prime}\right)_{\Omega_{h}}, \\
\left\langle{\widehat{e_{q}}}-c{\widehat{e_{u}}}^{c}, w n\right\rangle_{\partial \Omega_{h}} & =\left(e_{q}-c e_{u}, w^{\prime}\right)_{\Omega_{h}},
\end{aligned}
$$

for all $(v, w) \in \mathbb{V}_{h} \times \mathbb{W}_{h}$. Taking $(v, w)=\left(Z_{h}, \zeta_{h}\right)$ and subtracting the second equation from the first, we obtain

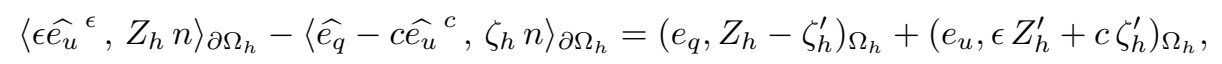

and using the definition of $(Z, \zeta)$,

$$
\begin{aligned}
\left\langle\epsilon{\widehat{e_{u}}}^{\epsilon}, Z_{h} n\right\rangle_{\partial \Omega_{h}}-\left\langle{\widehat{e_{q}}}-c{\widehat{e_{u}}}^{c}, \zeta_{h} n\right\rangle_{\partial \Omega_{h}}= & \left(e_{q},\left(Z_{h}-Z\right)-\left(\zeta_{h}-\zeta\right)^{\prime}\right)_{\Omega_{h}} \\
& +\left(e_{u}, \epsilon\left(Z_{h}-Z\right)^{\prime}+c\left(\zeta_{h}-\zeta\right)^{\prime}\right)_{\Omega_{h}} .
\end{aligned}
$$

The result follows by noting that

$$
\begin{aligned}
\left\langle\epsilon{\widehat{e_{u}}}^{\epsilon}, Z_{h} n\right\rangle_{\partial \Omega_{h}} & =\left\langle 1, \llbracket \epsilon{\widehat{e_{u}}}^{\epsilon} Z: h n \rrbracket\right\rangle_{\mathscr{E}_{h}}, \\
\left\langle{\widehat{e_{q}}}-c{\widehat{e_{u}}}^{c}, \zeta_{h} n\right\rangle_{\partial \Omega_{h}} & =\left\langle 1, \llbracket\left({\widehat{e_{q}}}^{-c}{\widehat{e_{u}}}^{c}\right) \zeta_{h} n \rrbracket\right\rangle_{\mathscr{E}_{h}} .
\end{aligned}
$$

This completes the proof.

We are now ready to prove the error representation formulas.

Proof of Lemma 3.1. Since ${\widehat{e_{u}}}^{\epsilon}(0)={\widehat{e_{u}}}^{\epsilon}(1)=0$, Lemma 3.1 follows from Lemma 3.2 if we show that if we take $(Z, \zeta)=\left(\varphi_{x_{i}}^{\prime}, \varphi_{x_{i}}\right)$, we have

$$
\left\langle 1, \llbracket \epsilon{\widehat{e_{u}}}^{\epsilon} Z n \rrbracket\right\rangle_{\mathscr{E}_{h}}-\left\langle 1, \llbracket\left({\widehat{e_{q}}}^{-}-{\widehat{e_{u}}}^{c}\right) \zeta n \rrbracket\right\rangle_{\mathscr{E}_{h}}=e_{\mathrm{u}}\left(x_{i}\right),
$$

and that if we take $(Z, \zeta)=\left(\psi_{x_{i}}^{\prime}, \psi_{x_{i}}\right)$,

$$
\left\langle 1, \llbracket \epsilon{\widehat{e_{u}}}^{\epsilon} Z n \rrbracket\right\rangle_{\mathscr{E}_{h}}-\left\langle 1, \llbracket\left(\widehat{e_{q}}-c{\widehat{e_{u}}}^{c}\right) \zeta n \rrbracket\right\rangle_{\mathscr{E}_{h}}=e_{\mathrm{flx}}\left(x_{i}\right)
$$

We only prove the first identity since the proof of the second is similar.

If $x_{i} \in \partial \Omega$, by the definition of the Green's function, (2.4) and (2.7), $\varphi_{x_{i}} \equiv 0$ and the identity follows since $\widehat{e_{\mathrm{u}}}\left(x_{i}\right)=0$. Assume now that $x_{i}$ is an interior node. Then

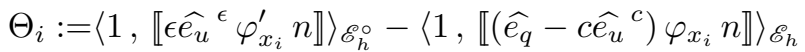

$$
\begin{aligned}
& =\left\langle 1, \llbracket \epsilon \widehat{e}_{u}{ }^{\epsilon} \varphi_{x_{i}}^{\prime} n \rrbracket\right\rangle_{\mathscr{E}_{h}^{\circ}}-\left\langle 1, \llbracket\left({\widehat{e_{q}}}^{-c}{\widehat{e_{u}}}^{c}\right) \varphi_{x_{i}} n \rrbracket\right\rangle_{\mathscr{E}_{h}^{\circ}}, \quad \text { since } \varphi_{x_{i}}=0 \text { at } \partial \Omega \\
& =\left\langle 1, \epsilon\left\{\left\{{\widehat{e_{u}}}^{\epsilon}\right\} \llbracket \llbracket \varphi_{x_{i}}^{\prime} n \rrbracket+\epsilon \llbracket \widehat{e_{u}}{ }^{\epsilon} n \rrbracket\left\{\left\{\varphi_{x_{i}}^{\prime}\right\}\right\rangle_{\mathscr{E}_{h}^{\circ}}\right.\right.
\end{aligned}
$$

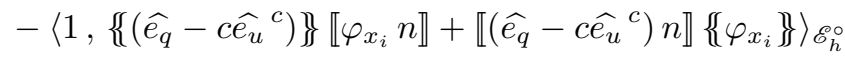

$$
\begin{aligned}
& =\left\{\left\{{\widehat{e_{u}}}^{\epsilon}\right\}\right\}\left(x_{i}\right)+\left\langle 1, \epsilon \llbracket{\widehat{e_{u}}}^{\epsilon} n \rrbracket\left\{\left\{\varphi_{x_{i}}^{\prime}\right\}\right\}-\llbracket\left({\widehat{e_{q}}}-c{\widehat{e_{u}}}^{c}\right) n \rrbracket\left\{\left\{\varphi_{x_{i}}\right\}\right\}\right\rangle_{\mathscr{E}_{h}^{\circ}} \text {, }
\end{aligned}
$$

by the definition of the Green's function $\varphi_{x_{i}},(2.4)$. Finally, by the definition of $e_{\mathrm{u}}\left(x_{i}\right),(2.3)$, we get that $\Theta_{i}=e_{\mathrm{u}}\left(x_{i}\right)$. This completes the proof. 
3.2. Proof of the main result, Theorem 2.1. The proof of the theorem follows from the error representation result, Lemma 3.1 and the approximation properties of a projection operator we introduce below.

Since $p=\min \left\{\ell_{\mathbb{V}_{h}}, \ell_{\mathbb{W}_{h}}\right\} \geq 1$, we can take $\left(Z_{h}, \zeta_{h}\right)=\left(\pi \varphi_{x_{i}}^{\prime}, \pi \varphi_{x_{i}}\right)$, in the first identity of Lemma 3.1, where $\pi \phi$, for any $\phi \in H^{1}\left(\Omega_{h}\right)$, is a function in $X_{h}^{p}$ defined on the element $I_{j}=\left(x_{j-1}, x_{j}\right)$ by

$$
\begin{aligned}
& (\pi \phi-\phi, v)_{I_{j}}=0 \quad \forall v \in P^{p-2}\left(I_{j}\right), \text { if } p>1, \\
& (\pi \phi)\left(x_{j}^{-}\right)=\phi\left(x_{j}^{-}\right), \quad(\pi \phi)\left(x_{j-1}^{+}\right)=\phi\left(x_{j-1}^{+}\right) .
\end{aligned}
$$

We readily obtain

$$
\begin{aligned}
e_{\mathrm{u}}\left(x_{i}\right)= & \left(e_{q},\left(\pi \varphi_{x_{i}}^{\prime}-\varphi_{x_{i}}^{\prime}\right)-\left(\pi \varphi_{x_{i}}-\varphi_{x_{i}}\right)^{\prime}\right)_{\Omega_{h}} \\
& +\left(e_{u}, \epsilon\left(\pi \varphi_{x_{i}}^{\prime}-\varphi_{x_{i}}^{\prime}\right)^{\prime}+c\left(\pi \varphi_{x_{i}}-\varphi_{x_{i}}\right)^{\prime}\right)_{\Omega_{h}} .
\end{aligned}
$$

This implies

$$
\begin{aligned}
\left|e_{\mathrm{u}}\left(x_{i}\right)\right| \leq & \left\|e_{q}\right\|_{0, \Omega_{h}}\left(\left\|\pi \varphi_{x_{i}}^{\prime}-\varphi_{x_{i}}^{\prime}\right\|_{0, \Omega_{h}}+\left\|\left(\pi \varphi_{x_{i}}-\varphi_{x_{i}}\right)^{\prime}\right\|_{0, \Omega_{h}}\right) \\
& +\left\|e_{u}\right\|_{0, \Omega_{h}}\left(\epsilon\left\|\left(\pi \varphi_{x_{i}}^{\prime}-\varphi_{x_{i}}^{\prime}\right)^{\prime}\right\|_{0, \Omega_{h}}+c\left\|\left(\pi \varphi_{x_{i}}-\varphi_{x_{i}}\right)^{\prime}\right\|_{0, \Omega_{h}}\right),
\end{aligned}
$$

and by the approximation properties of the operator $\pi$, namely,

$$
|\pi w-w|_{0, I_{j}}+\frac{h_{j}}{p}\left|(\pi w-w)^{\prime}\right|_{0, I_{j}} \leq C_{s}\left(\frac{h_{j}^{\min (s, p)+1}}{p^{s+1}}\right)|w|_{s+1, I_{j}}
$$

where the constant $C_{s}$ only depends on $s$ (see [27]), we get

$$
\begin{aligned}
\left|e_{\mathrm{u}}\left(x_{i}\right)\right| \leq & C_{s}\left\|e_{q}\right\|_{0, \Omega_{h}}\left(2\left|\varphi_{x_{i}}\right|_{s+1, \Omega_{h}}\right)\left(\frac{h_{j}^{\min (s, p)}}{p^{s}}\right) \\
& +C_{s}\left\|e_{u}\right\|_{0, \Omega_{h}}\left(\epsilon\left|\varphi_{x_{i}}\right|_{s+2, \Omega_{h}}+c\left|\varphi_{x_{i}}\right|_{s+1, \Omega_{h}}\right)\left(\frac{h_{j}^{\min (s, p)}}{p^{s}}\right) .
\end{aligned}
$$

Finally, by the definition of the Green's function $\varphi_{x_{i}}(2.4)$,

$$
\left|e_{\mathrm{u}}\left(x_{i}\right)\right| \leq 2 C_{s}\left(\left\|e_{q}\right\|_{0, \Omega_{h}}+c\left\|e_{u}\right\|_{0, \Omega_{h}}\right)\left(\frac{h_{j}^{\min (s, p)}}{p^{s}}\right)\left|\varphi_{x_{i}}\right|_{s+1, \Omega_{h}},
$$

and the first inequality of Theorem 2.1 follows. The other inequality can be obtained in the same way.

This completes the proof of Theorem 2.1.

3.3. Proof of the estimate for the md-DG methods, Theorem 2.6. To prove Theorem 2.6, we proceed as we did in the proof of Theorem 2.1. We begin by deriving suitable expressions for the numerical traces ${\widehat{e_{u}}}^{\epsilon}\left(x_{i}\right)$ and $\left(\widehat{e_{q}}-c{\widehat{e_{u}}}^{c}\right)\left(x_{i}\right)$. 
Lemma 3.3. For any node $x_{i}$, we have

$$
\begin{aligned}
\widehat{e_{u}}{ }^{\epsilon}\left(x_{i}\right)= & \epsilon \pi^{-} e_{u}\left(1^{-}\right)\left(\pi^{+} \varphi_{x_{i}}^{\prime}-\varphi_{x_{i}}^{\prime}\right)\left(1^{-}\right) \\
& +\left\langle c \llbracket \pi^{-} e_{u} n \rrbracket, \llbracket\left(\pi^{-} \varphi_{x_{i}}-\varphi_{x_{i}}\right) n \rrbracket\right\rangle_{\mathscr{E}_{h} \backslash\{1\}} \\
& +\left(q-\pi^{+} q,\left(\pi^{+} \varphi_{x_{i}}^{\prime}-\varphi_{x_{i}}^{\prime}\right)-\left(\pi^{-} \varphi_{x_{i}}-\varphi_{x_{i}}\right)^{\prime}\right)_{\Omega_{h}} \\
& +\left(\pi^{+} e_{q}, \pi^{+} \varphi_{x_{i}}^{\prime}-\varphi_{x_{i}}^{\prime}\right)_{\Omega_{h}}
\end{aligned}
$$

and

$$
\begin{aligned}
\left(\widehat{e_{q}}-c{\widehat{e_{u}}}^{c}\right)\left(x_{i}\right)= & \epsilon \pi^{-} e_{u}\left(1^{-}\right)\left(\pi^{+} \psi_{x_{i}}^{\prime}-\psi_{x_{i}}^{\prime}\right)\left(1^{-}\right) \\
& +\left\langle c \llbracket \pi^{-} e_{u} n \rrbracket \llbracket\left(\pi^{-} \psi_{x_{i}}-\psi_{x_{i}}\right) n \rrbracket\right\rangle_{\mathscr{E}_{h} \backslash\{1\}} \\
& +\left(q-\pi^{+} q,\left(\pi^{+} \psi_{x_{i}}^{\prime}-\psi_{x_{i}}^{\prime}\right)-\left(\pi^{-} \psi_{x_{i}}-\psi_{x_{i}}\right)^{\prime}\right)_{\Omega_{h}} \\
& +\left(\pi^{+} e_{q}, \pi^{+} \psi_{x_{i}}^{\prime}-\psi_{x_{i}}^{\prime}\right)_{\Omega_{h}} .
\end{aligned}
$$

Proof. We only prove the first identity, as the proof of the second is similar. From the error representation result, Lemma 3.1 with $\left(Z_{h}, \zeta_{h}\right)=\left(\pi^{+} \varphi_{x_{i}}^{\prime}, \pi^{-} \varphi_{x_{i}}\right)$, we have the expression

$$
\begin{aligned}
e_{\mathrm{u}}\left(x_{i}\right)= & \left\langle 1, \llbracket \epsilon{\widehat{e_{u}}}^{\epsilon}\left(\varphi_{x_{i}}^{\prime}-\pi^{+} \varphi_{x_{i}}^{\prime}\right) n \rrbracket\right\rangle_{\mathscr{E}_{h}^{\circ}} \\
& -\left\langle 1, \llbracket\left(\widehat{e_{q}}-c{\widehat{e_{u}}}^{c}\right)\left(\varphi_{x_{i}}-\pi^{-} \varphi_{x_{i}}\right) n \rrbracket\right\rangle_{\mathscr{E}_{h}} \\
& +\left(e_{q},\left(\pi^{+} \varphi_{x_{i}}^{\prime}-\varphi_{x_{i}}^{\prime}\right)-\left(\pi^{-} \varphi_{x_{i}}-\varphi_{x_{i}}\right)^{\prime}\right)_{\Omega_{h}} \\
& +\left(e_{u}, \epsilon\left(\pi^{+} \varphi_{x_{i}}^{\prime}-\varphi_{x_{i}}^{\prime}\right)^{\prime}+c\left(\pi^{-} \varphi_{x_{i}}-\varphi_{x_{i}}\right)^{\prime}\right)_{\Omega_{h}},
\end{aligned}
$$

which, after simple algebraic manipulations, can be written as

$$
e_{\mathrm{u}}\left(x_{i}\right)=T_{1}+T_{2}+T_{3}+T_{4},
$$

where

$$
\begin{aligned}
T_{1}= & \left\langle 1, \llbracket \epsilon{\widehat{e_{u}}}^{\epsilon}\left(\varphi_{x_{i}}^{\prime}-\pi^{+} \varphi_{x_{i}}^{\prime}\right) n \rrbracket\right\rangle_{\mathscr{E}_{h}^{\circ}}+\left(\pi^{-} e_{u}, \epsilon\left(\pi^{+} \varphi_{x_{i}}^{\prime}-\varphi_{x_{i}}^{\prime}\right)^{\prime}\right)_{\Omega_{h}}, \\
T_{2}= & -\left\langle 1, \llbracket\left({\widehat{e_{q}}}^{c}-c{\widehat{e_{u}}}^{c}\right)\left(\varphi_{x_{i}}-\pi^{-} \varphi_{x_{i}}\right) n \rrbracket\right\rangle_{\mathscr{E}_{h}} \\
& +\left(\left(-\epsilon \pi^{+} e_{q}+c \pi^{-} e_{u}\right),\left(\pi^{-} \varphi_{x_{i}}-\varphi_{x_{i}}\right)^{\prime}\right)_{\Omega_{h}}, \\
T_{3}= & \left(e_{q}-\pi^{+} e_{q},\left(\pi^{+} \varphi_{x_{i}}^{\prime}-\varphi_{x_{i}}^{\prime}\right)-\left(\pi^{-} \varphi_{x_{i}}-\varphi_{x_{i}}\right)^{\prime}\right)_{\Omega_{h}}+\left(\pi^{+} e_{q},\left(\pi^{+} \varphi_{x_{i}}^{\prime}-\varphi_{x_{i}}^{\prime}\right)\right)_{\Omega_{h}}, \\
T_{4}= & \left(e_{u}-\pi^{-} e_{u}, \epsilon\left(\pi^{+} \varphi_{x_{i}}^{\prime}-\varphi_{x_{i}}^{\prime}\right)^{\prime}+c\left(\pi^{-} \varphi_{x_{i}}-\varphi_{x_{i}}\right)^{\prime}\right)_{\Omega_{h}} .
\end{aligned}
$$

Let us work on the first term. Integrating by parts, we get

$$
\begin{aligned}
T_{1}= & \left\langle 1, \llbracket \epsilon\left({\widehat{e_{u}}}^{\epsilon}-\pi^{-} e_{u}\right)\left(\varphi_{x_{i}}^{\prime}-\pi^{+} \varphi_{x_{i}}^{\prime}\right) n \rrbracket\right\rangle_{\mathscr{E}_{h}^{\circ}}-\left\langle 1, \llbracket \epsilon \pi^{-} e_{u}\left(\varphi_{x_{i}}^{\prime}-\pi^{+} \varphi_{x_{i}}^{\prime}\right) n \rrbracket\right\rangle_{\partial \Omega} \\
& -\left(\epsilon\left(\pi^{-} e_{u}\right)^{\prime}, \pi^{+} \varphi_{x_{i}}^{\prime}-\varphi_{x_{i}}^{\prime}\right)_{\Omega_{h}} \\
= & \epsilon \pi^{-} e_{u}\left(1^{-}\right)\left(\varphi_{x_{i}}^{\prime}-\pi^{+} \varphi_{x_{i}}^{\prime}\right)\left(1^{-}\right),
\end{aligned}
$$

by the definition of the projection operators $\pi^{ \pm}(2.11)$ and the numerical trace $\widehat{u}_{h}^{\epsilon}$.

We deal with the second term in a similar way. Thus, integrating by parts, we get

$$
\begin{aligned}
T_{2}= & -\left\langle 1, \llbracket\left(\left(\widehat{e_{q}}-\pi^{+} e_{q}\right)-c\left({\widehat{e_{u}}}^{c}-\pi^{-} e_{u}\right)\right)\left(\varphi_{x_{i}}-\pi^{-} \varphi_{x_{i}}\right) n \rrbracket\right\rangle_{\mathscr{E}_{h}} \\
& -\left(\left(-\epsilon \pi^{+} e_{q}+c \pi^{-} e_{u}\right)^{\prime},\left(\pi^{-} \varphi_{x_{i}}-\varphi_{x_{i}}\right)\right)_{\Omega_{h}} \\
= & \left\langle 1, \llbracket c\left({\widehat{e_{u}}}^{c}-\pi^{-} e_{u}\right)\left(\varphi_{x_{i}}-\pi^{-} \varphi_{x_{i}}\right) n \rrbracket\right\rangle_{\mathscr{E}_{h}},
\end{aligned}
$$


by the definition of the projection operators $\pi^{ \pm}(2.11)$ and the numerical trace $\widehat{q_{h}}$. Since, for interior nodes $x_{i}$,

$$
\begin{aligned}
& \llbracket c\left({\widehat{e_{u}}}^{c}-\pi^{-} e_{u}\right)\left(\varphi_{x_{i}}-\pi^{-} \varphi_{x_{i}}\right) n \rrbracket\left(x_{i}\right) \\
& \quad=-c\left(\pi^{-} e_{u}\left(x_{i}^{-}\right)-\pi^{-} e_{u}\left(x_{i}^{+}\right)\right)\left(\varphi_{x_{i}}-\pi^{-} \varphi_{x_{i}}\right)\left(x_{i}^{+}\right) \\
& \quad=c \llbracket \pi^{-} e_{u} n \rrbracket \llbracket\left(\varphi_{x_{i}}-\pi^{-} \varphi_{x_{i}}\right) n \rrbracket\left(x_{i}\right)
\end{aligned}
$$

and

$$
\begin{aligned}
\llbracket c\left({\widehat{e_{u}}}^{c}-\pi^{-} e_{u}\right)\left(\varphi_{x_{i}}-\pi^{-} \varphi_{x_{i}}\right) n \rrbracket(0) & =c \pi^{-} e_{u}\left(0^{+}\right)\left(\varphi_{x_{i}}-\pi^{-} \varphi_{x_{i}}\right)\left(0^{+}\right) \\
& =c \llbracket \pi^{-} e_{u} n \rrbracket \llbracket\left(\varphi_{x_{i}}-\pi^{-} \varphi_{x_{i}}\right) n \rrbracket(0), \\
\llbracket c\left({\widehat{e_{u}}}^{c}-\pi^{-} e_{u}\right)\left(\varphi_{x_{i}}-\pi^{-} \varphi_{x_{i}}\right) n \rrbracket(1) & =0,
\end{aligned}
$$

again by the definition of the projection operators $\pi^{ \pm},(2.11)$, and the numerical trace $\widehat{u}_{h}^{c}$, we get that

$$
T_{2}=\left\langle c \llbracket \pi^{-} e_{u} n \rrbracket, \llbracket\left(\pi^{-} \varphi_{x_{i}}-\varphi_{x_{i}}\right) n \rrbracket\right\rangle_{\mathscr{E}_{h} \backslash\{1\}} .
$$

For the term $T_{3}$, we simply use the fact that $e_{q}-\pi^{+} e_{q}=q-\pi^{+} q$ to write $T_{3}=\left(q-\pi^{+} q,\left(\pi^{+} \varphi_{x_{i}}^{\prime}-\varphi_{x_{i}}^{\prime}\right)-\left(\pi^{-} \varphi_{x_{i}}-\varphi_{x_{i}}\right)^{\prime}\right)_{\Omega_{h}}+\left(\pi^{+} e_{q},\left(\pi^{+} \varphi_{x_{i}}^{\prime}-\varphi_{x_{i}}^{\prime}\right)\right)_{\Omega_{h}}$.

Finally, by the definition of the Green's function $\varphi_{x_{i}},(2.4)$,

$$
T_{4}=\left(e_{u}-\pi^{-} e_{u},\left(\epsilon \pi^{+} \varphi_{x_{i}}^{\prime}+c \pi^{-} \varphi_{x_{i}}\right)^{\prime}\right)_{\Omega_{h}}=0,
$$

by the definition of the projection operators $\pi^{ \pm},(2.11)$. This completes the proof.

We are now ready to prove Theorem 2.6. We only prove the first inequality since the second can be obtained in a similar way. Thus, from the above lemma and the definition of the energy seminorm $|(\cdot, \cdot)|_{\mathcal{A}_{h}}(2.10)$,

$$
\begin{aligned}
\left|{\widehat{e_{u}}}^{\epsilon}\left(x_{i}\right)\right| \leq & \left\|q-\pi^{+} q\right\|_{0, \Omega_{h}}\left(\left\|\pi^{+} \varphi_{x_{i}}^{\prime}-\varphi_{x_{i}}^{\prime}\right\|_{0, \Omega_{h}}+\left\|\left(\pi^{-} \varphi_{x_{i}}-\varphi_{x_{i}}\right)^{\prime}\right\|_{0, \Omega_{h}}\right) \\
& +\left\|\pi^{+} e_{q}\right\|_{0, \Omega_{h}}\left\|\pi^{+} \varphi_{x_{i}}^{\prime}-\varphi_{x_{i}}^{\prime}\right\|_{0, \Omega_{h}} \\
& +\left(\left\langle c, \llbracket \pi^{-} e_{u} n \rrbracket^{2}\right\rangle_{\mathscr{E}_{h} \backslash\{1\}}\left\langle c, \llbracket\left(\pi^{-} \varphi_{x_{i}}-\varphi_{x_{i}}\right) n \rrbracket^{2}\right\rangle_{\mathscr{E}_{h} \backslash\{1\}}\right)^{1 / 2} \\
& +\epsilon\left|\pi^{-} e_{u}\left(1^{-}\right) \|\left(\pi^{+} \varphi_{x_{i}}^{\prime}-\varphi_{x_{i}}^{\prime}\right)\left(1^{-}\right)\right| \\
\leq & \left\|q-\pi^{+} q\right\|_{0, \Omega_{h}}\left(\left\|\pi^{+} \varphi_{x_{i}}^{\prime}-\varphi_{x_{i}}^{\prime}\right\|_{0, \Omega_{h}}+\left\|\left(\pi^{-} \varphi_{x_{i}}-\varphi_{x_{i}}\right)^{\prime}\right\|_{0, \Omega_{h}}\right) \\
& +\left|\left(\pi^{+} e_{q}, \pi^{-} e_{u}\right)\right|_{\mathcal{A}_{h}} \Theta_{i},
\end{aligned}
$$

where

$$
\Theta_{i}^{2}=\epsilon\left\|\pi^{+} \varphi_{x_{i}}^{\prime}-\varphi_{x_{i}}^{\prime}\right\|_{0, \Omega_{h}}^{2}+\left\langle c, \llbracket\left(\pi^{-} \varphi_{x_{i}}-\varphi_{x_{i}}\right) n \rrbracket^{2}\right\rangle_{\mathscr{E}_{h} \backslash\{1\}}+\frac{\epsilon^{2}}{\alpha}\left|\left(\pi^{+} \varphi_{x_{i}}^{\prime}-\varphi_{x_{i}}^{\prime}\right)\left(1^{-}\right)\right|^{2} .
$$

Finally, using the approximation properties of the operators $\pi^{ \pm}$, namely,

$$
\begin{gathered}
\left|\pi^{ \pm} w-w\right|_{0, I_{j}}+\frac{h_{j}}{p}\left|\left(\pi^{ \pm} w-w\right)^{\prime}\right|_{0, I_{j}} \leq C_{s}\left(\frac{h_{j}^{\min (s, p)+1}}{p^{s+1}}\right)|w|_{s+1, I_{j}}, \\
\left|\left(\pi^{+} w-w\right)\left(x_{j}\right)\right|+\left|\left(\pi^{-} w-w\right)\left(x_{j-1}\right)\right| \leq C_{s}\left(\frac{h_{j}^{\min (s, p)+1 / 2}}{p^{s+1 / 2}}\right)|w|_{s+1, I_{j}},
\end{gathered}
$$


where $C_{s}$ depends only on $s$ (see [26]; see also [14]), and using the definition of the Green's function $\varphi_{x_{i}}$, we easily get that

$$
\left\|\pi^{+} \varphi_{x_{i}}^{\prime}-\varphi_{x_{i}}^{\prime}\right\|_{0, \Omega_{h}}+\left\|\left(\pi^{-} \varphi_{x_{i}}-\varphi_{x_{i}}\right)^{\prime}\right\|_{0, \Omega_{h}} \leq 2 C_{s}\left(\frac{h_{j}^{\min (s, p)}}{p^{s}}\right)\left|\varphi_{x_{i}}\right|_{s+1, \Omega_{h}}
$$

and that

$$
\left|\Theta_{i}\right| \leq 3 C_{s} \sqrt{\epsilon}\left(\frac{h_{j}^{\min (s, p)}}{p^{s}}\right)\left|\varphi_{x_{i}}\right|_{s+1, \Omega_{h}} .
$$

As a consequence

$$
\left|{\widehat{e_{u}}}^{\epsilon}\left(x_{i}\right)\right| \leq 3 C_{s}\left(\left\|q-\pi^{+} q\right\|_{0, \Omega_{h}}+\sqrt{\epsilon}\left|\left(\pi^{+} e_{q}, \pi^{-} e_{u}\right)\right|_{\mathcal{A}_{h}}\right)\left(\frac{h_{j}^{\min (s, p)}}{p^{s}}\right)\left|\varphi_{x_{i}}\right|_{s+1, \Omega_{h}},
$$

and the first inequality of Theorem 2.6 follows. This completes the proof of Theorem 2.6 .

\section{Numerical RESUlts}

In this section, we numerically verify the sharpness of our theoretical findings and explore some situations not covered by them. In all the experiments, we take $\epsilon=c=1$. The function $f$ is chosen so that the exact solution is $u(x)=e^{x} \sin (\pi x)$, except in Section 4.5 for which we take $u(x)=x^{7 / 2}$.

We display the history of convergence of our methods in several tables. Therein, $p$ indicates the polynomial degree used to define the method. A uniform mesh with $2^{i}$ elements is called the mesh " $i$ "; nonuniform meshes are used in the very last numerical experiment. We also display numerical rates of convergence which are computed as follows. Let $e(j)$ denote the error of the approximation computed on the mesh $j$. Then the approximate order of convergence, $r_{i}$, is defined by

$$
r_{i}:=\frac{\log \left(\frac{e(i-1)}{e(i)}\right)}{\log 2} .
$$

Finally, we define $\left\|{\widehat{e_{u}}}^{\epsilon}\right\|_{\infty}:=\max _{1 \leq j \leq N}\left|\left(u-\widehat{u}_{h}^{\epsilon}\right)\left(x_{j}\right)\right|$. The quantity $\left\|\widehat{e_{q}}-c{\widehat{e_{u}}}^{c}\right\|_{\infty}$ is defined in a similar way.

TABLE 5. History of convergence of the B.Z. method with $\alpha=\left(\frac{p}{h}\right)^{p+1}$.

\begin{tabular}{cccccccccc}
\hline & \multicolumn{4}{c}{$\left\|\left(e_{u}, e_{q}\right)\right\|_{c, \Omega_{h}}$} & \multicolumn{2}{c}{$\| u-\left\{\left\{u_{h}\right\} \|_{\infty}\right.$} & \multicolumn{2}{c}{$\left\|\widehat{e_{q}}-c{\widehat{e_{u}}}^{c}\right\|_{\infty}$} & \multicolumn{2}{c}{$\left(\sum h^{-1} \llbracket u_{h} n \rrbracket^{2}\right)^{1 / 2}$} \\
$\mathrm{p}$ mesh & error & order & error & order & error & order & error & order \\
\hline \hline & 4 & $3.79 \mathrm{E}-01$ & 1.06 & $9.33 \mathrm{E}-02$ & 0.79 & $6.04 \mathrm{E}-02$ & 0.97 & $2.27 \mathrm{E}-01$ & 0.85 \\
1 & 5 & $1.83 \mathrm{E}-01$ & 1.05 & $4.96 \mathrm{E}-02$ & 0.91 & $3.08 \mathrm{E}-02$ & 0.97 & $1.19 \mathrm{E}-01$ & 0.93 \\
& 6 & $8.97 \mathrm{E}-02$ & 1.03 & $2.55 \mathrm{E}-02$ & 0.96 & $1.55 \mathrm{E}-02$ & 0.98 & $6.07 \mathrm{E}-02$ & 0.97 \\
& 7 & $4.43 \mathrm{E}-02$ & 1.02 & $1.29 \mathrm{E}-02$ & 0.98 & $7.82 \mathrm{E}-03$ & 0.99 & $3.07 \mathrm{E}-02$ & 0.98 \\
\hline & 4 & $7.29 \mathrm{E}-03$ & 2.16 & $7.25 \mathrm{E}-04$ & 1.79 & $4.91 \mathrm{E}-04$ & 1.99 & $1.78 \mathrm{E}-03$ & 1.86 \\
2 & 5 & $1.69 \mathrm{E}-03$ & 2.11 & $1.93 \mathrm{E}-04$ & 1.91 & $1.23 \mathrm{E}-04$ & 2.00 & $4.65 \mathrm{E}-04$ & 1.94 \\
& 6 & $4.01 \mathrm{E}-04$ & 2.07 & $4.97 \mathrm{E}-05$ & 1.96 & $3.07 \mathrm{E}-05$ & 2.00 & $1.19 \mathrm{E}-04$ & 1.97 \\
& 7 & $9.77 \mathrm{E}-05$ & 2.04 & $1.26 \mathrm{E}-05$ & 1.98 & $7.68 \mathrm{E}-06$ & 2.00 & $3.00 \mathrm{E}-05$ & 1.98 \\
\hline & 4 & $1.39 \mathrm{E}-04$ & 3.07 & $4.48 \mathrm{E}-06$ & 2.79 & $3.03 \mathrm{E}-06$ & 2.99 & $1.10 \mathrm{E}-05$ & 2.86 \\
3 & 5 & $1.70 \mathrm{E}-05$ & 3.04 & $5.96 \mathrm{E}-07$ & 2.91 & $3.79 \mathrm{E}-07$ & 3.00 & $1.43 \mathrm{E}-06$ & 2.94 \\
& 6 & $2.09 \mathrm{E}-06$ & 3.02 & $7.67 \mathrm{E}-08$ & 2.96 & $4.74 \mathrm{E}-08$ & 3.00 & $1.83 \mathrm{E}-07$ & 2.97 \\
& 7 & $2.60 \mathrm{E}-07$ & 3.01 & $9.72 \mathrm{E}-09$ & 2.98 & $5.93 \mathrm{E}-09$ & 3.00 & $2.31 \mathrm{E}-08$ & 2.98 \\
\hline & 4 & $1.43 \mathrm{E}-06$ & 4.02 & $2.21 \mathrm{E}-08$ & 3.79 & $1.50 \mathrm{E}-08$ & 3.99 & $5.43 \mathrm{E}-08$ & 3.86 \\
4 & 5 & $8.91 \mathrm{E}-08$ & 4.00 & $1.47 \mathrm{E}-09$ & 3.91 & $9.43 \mathrm{E}-10$ & 3.99 & $3.55 \mathrm{E}-09$ & 3.94 \\
& 6 & $5.56 \mathrm{E}-09$ & 4.00 & $9.47 \mathrm{E}-11$ & 3.96 & $5.90 \mathrm{E}-11$ & 4.00 & $2.26 \mathrm{E}-10$ & 3.97 \\
& 7 & $3.48 \mathrm{E}-10$ & 4.00 & $6.01 \mathrm{E}-12$ & 3.98 & $3.68 \mathrm{E}-12$ & 4.00 & $1.43 \mathrm{E}-11$ & 3.98 \\
\hline
\end{tabular}


TABLE 6. History of convergence of the B.O. method.

\begin{tabular}{cccccccccc}
\hline & \multicolumn{4}{c}{$\left\|\left(e_{u}, e_{q}\right)\right\|_{c, \Omega_{h}}$} & \multicolumn{2}{c}{$\| u-\left\{\left\{u_{h}\right\} \|_{\infty}\right.$} & \multicolumn{2}{c}{$\left\|\widehat{e_{q}}-c{\widehat{e_{u}}}^{c}\right\|_{\infty}$} & \multicolumn{3}{c}{$\left(\sum h^{-1} \llbracket u_{h} n \rrbracket^{2}\right)^{1 / 2}$} \\
$\mathrm{p}$ mesh & error & order & error & order & error & order & error & order \\
\hline \hline & 4 & $2.50 \mathrm{E}+00$ & 1.15 & $1.75 \mathrm{E}-01$ & 1.22 & $2.82 \mathrm{E}-01$ & 1.76 & $1.13 \mathrm{E}+00$ & 1.00 \\
1 & 5 & $1.28 \mathrm{E}+00$ & 0.96 & $6.74 \mathrm{E}-02$ & 1.38 & $9.21 \mathrm{E}-02$ & 1.61 & $6.25 \mathrm{E}-01$ & 0.85 \\
& 6 & $7.01 \mathrm{E}-01$ & 0.87 & $2.46 \mathrm{E}-02$ & 1.46 & $3.11 \mathrm{E}-02$ & 1.57 & $3.60 \mathrm{E}-01$ & 0.80 \\
& 7 & $3.94 \mathrm{E}-01$ & 0.83 & $8.74 \mathrm{E}-03$ & 1.49 & $1.06 \mathrm{E}-02$ & 1.55 & $2.10 \mathrm{E}-01$ & 0.78 \\
\hline & 4 & $3.83 \mathrm{E}-02$ & 1.86 & $6.04 \mathrm{E}-03$ & 1.60 & $1.78 \mathrm{E}-02$ & 1.87 & $1.12 \mathrm{E}-02$ & 1.82 \\
2 & 5 & $9.96 \mathrm{E}-03$ & 1.94 & $1.67 \mathrm{E}-03$ & 1.86 & $4.59 \mathrm{E}-03$ & 1.95 & $2.95 \mathrm{E}-03$ & 1.92 \\
& 6 & $2.53 \mathrm{E}-03$ & 1.98 & $4.35 \mathrm{E}-04$ & 1.94 & $1.16 \mathrm{E}-03$ & 1.98 & $7.54 \mathrm{E}-04$ & 1.97 \\
& 7 & $6.36 \mathrm{E}-04$ & 1.99 & $1.11 \mathrm{E}-04$ & 1.97 & $2.92 \mathrm{E}-04$ & 1.99 & $1.90 \mathrm{E}-04$ & 1.98 \\
\hline & 4 & $1.66 \mathrm{E}-04$ & 3.27 & $1.91 \mathrm{E}-06$ & 3.84 & $1.03 \mathrm{E}-05$ & 3.99 & $8.36 \mathrm{E}-06$ & 3.79 \\
3 & 5 & $1.85 \mathrm{E}-05$ & 3.17 & $1.41 \mathrm{E}-07$ & 3.76 & $6.45 \mathrm{E}-07$ & 4.00 & $6.64 \mathrm{E}-07$ & 3.65 \\
& 6 & $2.16 \mathrm{E}-06$ & 3.10 & $9.86 \mathrm{E}-09$ & 3.84 & $4.03 \mathrm{E}-08$ & 4.00 & $5.56 \mathrm{E}-08$ & 3.58 \\
& 7 & $2.61 \mathrm{E}-07$ & 3.05 & $6.54 \mathrm{E}-10$ & 3.91 & $2.52 \mathrm{E}-09$ & 4.00 & $4.78 \mathrm{E}-09$ & 3.54 \\
\hline & 4 & $3.81 \mathrm{E}-06$ & 3.97 & $1.90 \mathrm{E}-07$ & 3.84 & $1.17 \mathrm{E}-06$ & 3.95 & $6.92 \mathrm{E}-07$ & 3.96 \\
4 & 5 & $2.40 \mathrm{E}-07$ & 3.99 & $1.21 \mathrm{E}-08$ & 3.97 & $7.38 \mathrm{E}-08$ & 3.99 & $4.37 \mathrm{E}-08$ & 3.99 \\
& 6 & $1.50 \mathrm{E}-08$ & 4.00 & $7.60 \mathrm{E}-10$ & 3.99 & $4.63 \mathrm{E}-09$ & 4.00 & $2.74 \mathrm{E}-09$ & 4.00 \\
& 7 & $9.40 \mathrm{E}-10$ & 4.00 & $4.75 \mathrm{E}-11$ & 4.00 & $2.90 \mathrm{E}-10$ & 4.00 & $1.72 \mathrm{E}-10$ & 4.00 \\
\hline
\end{tabular}

TABLE 7. History of convergence of the NIPG method.

\begin{tabular}{|c|c|c|c|c|c|c|c|c|c|}
\hline \multirow[b]{2}{*}{$\mathrm{p}$} & \multirow[b]{2}{*}{ mesh } & \multicolumn{2}{|c|}{$\left\|\left(e_{u}, e_{q}\right)\right\|_{c, \Omega_{h}}$} & \multicolumn{2}{|c|}{$\| u-\left\{\left\{u_{h}\right\} \|_{\infty}\right.$} & \multicolumn{2}{|c|}{ 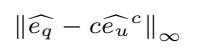 } & \multicolumn{2}{|c|}{$\left(\sum h^{-1} \llbracket u_{h} n \rrbracket^{2}\right)^{1 / 2}$} \\
\hline & & error & order & error & order & error & order & error & order \\
\hline \multirow{4}{*}{1} & 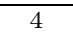 & $1.51 \mathrm{E}+00$ & $\overline{~ 1.34}$ & 8.89E-02 & 1.50 & $9.40 \mathrm{E}-02$ & 1.76 & $2.58 \mathrm{E}-01$ & 1.34 \\
\hline & 5 & $5.72 \mathrm{E}-01$ & 1.40 & $2.61 \mathrm{E}-02$ & 1.77 & $2.58 \mathrm{E}-02$ & 1.87 & $9.65 \mathrm{E}-02$ & 1.42 \\
\hline & 6 & $2.13 \mathrm{E}-01$ & 1.43 & $7.06 \mathrm{E}-03$ & 1.89 & $6.76 \mathrm{E}-03$ & 1.93 & $3.51 \mathrm{E}-02$ & 1.46 \\
\hline & 7 & 7.99E-02 & 1.41 & $1.83 \mathrm{E}-03$ & 1.94 & $1.73 \mathrm{E}-03$ & 1.96 & $1.26 \mathrm{E}-02$ & 1.48 \\
\hline \multirow{4}{*}{2} & 4 & $2.01 \mathrm{E}-02$ & 2.08 & $2.35 \mathrm{E}-03$ & 1.87 & $6.15 \mathrm{E}-03$ & 1.96 & $4.14 \mathrm{E}-03$ & 1.95 \\
\hline & 5 & $4.74 \mathrm{E}-03$ & 2.09 & $5.99 \mathrm{E}-04$ & 1.97 & $1.55 \mathrm{E}-03$ & 1.99 & $1.04 \mathrm{E}-03$ & 1.99 \\
\hline & 6 & $1.13 \mathrm{E}-03$ & 2.07 & $1.50 \mathrm{E}-04$ & 1.99 & $3.90 \mathrm{E}-04$ & 1.99 & $2.59 \mathrm{E}-04$ & 2.01 \\
\hline & 7 & $2.75 \mathrm{E}-04$ & 2.04 & $3.77 \mathrm{E}-05$ & 2.00 & $9.76 \mathrm{E}-05$ & 2.00 & $6.44 \mathrm{E}-05$ & 2.01 \\
\hline \multirow{4}{*}{3} & 4 & $1.85 \mathrm{E}-04$ & 3.32 & $1.84 \mathrm{E}-06$ & 3.78 & $7.70 \mathrm{E}-06$ & 3.99 & $6.82 \mathrm{E}-06$ & 3.73 \\
\hline & 5 & $1.98 \mathrm{E}-05$ & 3.22 & $1.34 \mathrm{E}-07$ & 3.78 & $4.82 \mathrm{E}-07$ & 4.00 & $5.57 \mathrm{E}-07$ & 3.62 \\
\hline & 6 & $2.25 \mathrm{E}-06$ & 3.14 & $9.16 \mathrm{E}-09$ & 3.87 & $3.01 \mathrm{E}-08$ & 4.00 & $4.74 \mathrm{E}-08$ & 3.55 \\
\hline & 7 & $2.67 \mathrm{E}-07$ & 3.08 & $6.02 \mathrm{E}-10$ & 3.93 & $1.88 \mathrm{E}-09$ & 4.00 & $4.11 \mathrm{E}-09$ & 3.53 \\
\hline \multirow{4}{*}{4} & 4 & $2.83 \mathrm{E}-06$ & 3.99 & $1.15 \mathrm{E}-07$ & 3.86 & $7.05 \mathrm{E}-07$ & 3.97 & $4.18 \mathrm{E}-07$ & 3.97 \\
\hline & 5 & $1.78 \mathrm{E}-07$ & 3.99 & 7.27E-09 & 3.98 & $4.44 \mathrm{E}-08$ & 3.99 & $2.63 \mathrm{E}-08$ & 3.99 \\
\hline & 6 & $1.11 \mathrm{E}-08$ & 4.00 & $4.56 \mathrm{E}-10$ & 3.99 & $2.78 \mathrm{E}-09$ & 4.00 & 1.65E-09 & 4.00 \\
\hline & 7 & $6.95 \mathrm{E}-10$ & 4.00 & $2.85 \mathrm{E}-11$ & 4.00 & $1.74 \mathrm{E}-10$ & 4.00 & $1.03 \mathrm{E}-10$ & 4.00 \\
\hline
\end{tabular}

Note that since the order of convergence of the numerical traces for many methods considered here is equal to or greater than $2 p+1$, we made use of the highprecision library provided by D. H. Bailey [6] to perform our numerical computations. This library allows one to carry out scientific computations at an arbitrary level of precision. In our numerical experiments, we performed the computations with 32-digit accuracy.

4.1. Methods with nonconservative numerical traces. We begin by considering the B.Z. method. If we take the penalization parameter $\alpha=p / h$, this method does not converge because its numerical trace $\widehat{q}_{h}$ is not consistent. In particular, the quantity $\left(\sum h^{-1} \llbracket u_{h} n \rrbracket^{2}\right)^{1 / 2}$ remains of order one as $h / p$ go to zero. An illustration of this phenomenon is given in Figure 1. Therein, the little triangles denote the values of the numerical trace $\widehat{u}_{h}^{\epsilon}$, which are two per interior node. The values of the numerical trace $\widehat{q}_{h}$ are denoted by little diamonds. In Table 5 , we show the performance of the method when we take $\alpha=(p / h)^{p+1}$. Note that since the numerical trace $\widehat{u}_{h}^{\epsilon}$ is not conservative, we cannot display the order of convergence for $\left\|\widehat{e_{u}}{ }^{\epsilon}\right\|_{\infty}$. However, a corollary to Theorem 2.1 predicts that both of the quantities 
$\left\{\left\{u_{h}\right\}\right\}$ and $\widehat{q}_{h}-c \widehat{u}_{h}$ converge with the order of convergence of $\left(\sum h^{-1} \llbracket u_{h} n \rrbracket\right)^{1 / 2}$. The results in Table 5 verify the prediction.

Similar results hold for the B.O. and NIPG methods, both of which have a nonconservative numerical trace $\widehat{u}_{h}^{\epsilon}$; see Tables 6 and 7 . These three methods have the same behavior in the case $c=0$.

4.2. Methods with conservative numerical traces. Let us begin by considering the modified B.Z. method, m-B.Z. The only difference between the B.Z. and the m-B.Z. methods concerns the numerical trace $\widehat{u}_{h}^{\epsilon}$ : For both methods such a trace is consistent, but it is conservative only for the m-B.Z. method. So, comparing the performances of these two methods gives us an idea of the relevance of having conservative numerical traces.

In Table 8 we display the numerical results for the m-B.Z. method $\alpha=p / h$. First, we see that, as a consequence of the lack of consistency of the numerical trace for $\widehat{q}_{h}$, the method does not always converge in $\|\cdot\|_{c, \Omega_{h}}$-norm. Nevertheless,

TABLE 8. History of convergence of the m-B.Z. method with $\alpha=\epsilon p / h$.

\begin{tabular}{cccccccccc}
\hline & & \multicolumn{2}{c}{$\left\|e_{u}\right\|_{0, \Omega_{h}}$} & \multicolumn{2}{c}{$\left\|e_{q}\right\|_{0, \Omega_{h}}$} & \multicolumn{2}{c}{$\left\|\widehat{e_{u}}\right\|_{\infty}$} & \multicolumn{2}{c}{$\left\|\widehat{e_{q}}-c{\widehat{e_{u}}}^{c}\right\|_{\infty}$} \\
$\mathrm{p}$ mesh & error & order & error & order & error & order & error & order \\
\hline \hline \multirow{2}{*}{1} & 4 & $8.87 \mathrm{E}-02$ & 1.18 & $2.02 \mathrm{E}+00$ & 0.54 & $7.18 \mathrm{E}-03$ & 1.81 & $1.50 \mathrm{E}-02$ & 1.95 \\
& 5 & $4.05 \mathrm{E}-02$ & 1.13 & $1.41 \mathrm{E}+00$ & 0.52 & $1.93 \mathrm{E}-03$ & 1.90 & $3.81 \mathrm{E}-03$ & 1.97 \\
& 6 & $1.92 \mathrm{E}-02$ & 1.08 & $9.91 \mathrm{E}-01$ & 0.51 & $5.01 \mathrm{E}-04$ & 1.95 & $9.61 \mathrm{E}-04$ & 1.99 \\
& 7 & $9.28 \mathrm{E}-03$ & 1.05 & $6.99 \mathrm{E}-01$ & 0.50 & $1.28 \mathrm{E}-04$ & 1.97 & $2.41 \mathrm{E}-04$ & 1.99 \\
\hline \multirow{2}{*}{2} & 4 & $4.24 \mathrm{E}-02$ & 1.16 & $5.13 \mathrm{E}+00$ & 0.12 & $2.98 \mathrm{E}-04$ & 2.11 & $1.60 \mathrm{E}-04$ & 1.94 \\
& 5 & $1.97 \mathrm{E}-02$ & 1.11 & $4.82 \mathrm{E}+00$ & 0.09 & $7.13 \mathrm{E}-05$ & 2.06 & $4.06 \mathrm{E}-05$ & 1.98 \\
& 6 & $9.41 \mathrm{E}-03$ & 1.06 & $4.64 \mathrm{E}+00$ & 0.05 & $1.74 \mathrm{E}-05$ & 2.03 & $1.02 \mathrm{E}-05$ & 1.99 \\
& 7 & $4.59 \mathrm{E}-03$ & 1.03 & $4.54 \mathrm{E}+00$ & 0.03 & $4.31 \mathrm{E}-06$ & 2.02 & $2.56 \mathrm{E}-06$ & 2.00 \\
\hline & 4 & $1.92 \mathrm{E}-02$ & 1.21 & $1.16 \mathrm{E}+00$ & 0.67 & $1.70 \mathrm{E}-07$ & 3.94 & $3.96 \mathrm{E}-07$ & 3.99 \\
& 5 & $8.75 \mathrm{E}-03$ & 1.13 & $7.64 \mathrm{E}-01$ & 0.60 & $1.09 \mathrm{E}-08$ & 3.96 & $2.49 \mathrm{E}-08$ & 3.99 \\
& 6 & $4.15 \mathrm{E}-03$ & 1.08 & $5.21 \mathrm{E}-01$ & 0.55 & $6.94 \mathrm{E}-10$ & 3.98 & $1.56 \mathrm{E}-09$ & 4.00 \\
& 7 & $2.01 \mathrm{E}-03$ & 1.04 & $3.62 \mathrm{E}-01$ & 0.53 & $4.38 \mathrm{E}-11$ & 3.99 & $9.75 \mathrm{E}-11$ & 4.00 \\
\hline & 4 & $1.41 \mathrm{E}-02$ & 1.18 & $3.47 \mathrm{E}+00$ & 0.13 & $4.18 \mathrm{E}-09$ & 4.12 & $2.23 \mathrm{E}-09$ & 3.91 \\
4 & 5 & $6.49 \mathrm{E}-03$ & 1.12 & $3.25 \mathrm{E}+00$ & 0.10 & $2.50 \mathrm{E}-10$ & 4.07 & $1.42 \mathrm{E}-10$ & 3.98 \\
& 6 & $3.09 \mathrm{E}-03$ & 1.07 & $3.12 \mathrm{E}+00$ & 0.06 & $1.52 \mathrm{E}-11$ & 4.03 & $8.91 \mathrm{E}-12$ & 3.99 \\
& 7 & $1.51 \mathrm{E}-03$ & 1.04 & $3.05 \mathrm{E}+00$ & 0.03 & $9.40 \mathrm{E}-13$ & 4.02 & $5.58 \mathrm{E}-13$ & 4.00 \\
\hline
\end{tabular}

TABLE 9. History of convergence of the m-B.Z. method with $\alpha=\epsilon\left(\frac{p}{h}\right)^{p+1}$.

\begin{tabular}{cccccccc}
\hline & \multicolumn{4}{c}{$\left\|\left(e_{u}, e_{q}\right)\right\|_{c, \Omega_{h}}$} & \multicolumn{2}{c}{$\left\|\widehat{e_{u}}\right\|_{\infty}$} & \multicolumn{2}{c}{$\left\|\widehat{e_{q}}-c \widehat{e_{u}}{ }^{c}\right\|_{\infty}$} \\
$\mathrm{p}$ mesh & error & order & error & order & error & order \\
\hline \hline \multirow{3}{*}{1} & 4 & $2.34 \mathrm{E}-01$ & 0.80 & $1.13 \mathrm{E}-03$ & 1.59 & $1.76 \mathrm{E}-03$ & 0.52 \\
& 5 & $1.27 \mathrm{E}-01$ & 0.88 & $3.21 \mathrm{E}-04$ & 1.81 & $5.83 \mathrm{E}-04$ & 1.59 \\
& 6 & $6.62 \mathrm{E}-02$ & 0.94 & $8.54 \mathrm{E}-05$ & 1.91 & $1.64 \mathrm{E}-04$ & 1.83 \\
& 7 & $3.38 \mathrm{E}-02$ & 0.97 & $2.20 \mathrm{E}-05$ & 1.96 & $4.32 \mathrm{E}-05$ & 1.92 \\
\hline \multirow{3}{*}{2} & 4 & $9.84 \mathrm{E}-03$ & 2.07 & $5.27 \mathrm{E}-07$ & 4.05 & $7.68 \mathrm{E}-07$ & 3.98 \\
& 5 & $2.38 \mathrm{E}-03$ & 2.05 & $3.19 \mathrm{E}-08$ & 4.04 & $4.81 \mathrm{E}-08$ & 4.00 \\
& 6 & $5.83 \mathrm{E}-04$ & 2.03 & $1.96 \mathrm{E}-09$ & 4.02 & $3.01 \mathrm{E}-09$ & 4.00 \\
& 7 & $1.44 \mathrm{E}-04$ & 2.01 & $1.22 \mathrm{E}-10$ & 4.01 & $1.88 \mathrm{E}-10$ & 4.00 \\
\hline \multirow{3}{*}{3} & 4 & $1.36 \mathrm{E}-04$ & 3.08 & $3.95 \mathrm{E}-11$ & 6.03 & $2.70 \mathrm{E}-11$ & 6.19 \\
& 5 & $1.66 \mathrm{E}-05$ & 3.04 & $6.08 \mathrm{E}-13$ & 6.02 & $3.93 \mathrm{E}-13$ & 6.10 \\
& 6 & $2.04 \mathrm{E}-06$ & 3.02 & $9.42 \mathrm{E}-15$ & 6.01 & $5.92 \mathrm{E}-15$ & 6.05 \\
& 7 & $2.53 \mathrm{E}-07$ & 3.01 & $1.47 \mathrm{E}-16$ & 6.01 & $9.08 \mathrm{E}-17$ & 6.03 \\
\hline \multirow{3}{*}{4} & 4 & $1.43 \mathrm{E}-06$ & 4.02 & $7.47 \mathrm{E}-16$ & 8.04 & $3.47 \mathrm{E}-15$ & 8.01 \\
& 5 & $8.86 \mathrm{E}-08$ & 4.01 & $2.89 \mathrm{E}-18$ & 8.02 & $1.44 \mathrm{E}-17$ & 7.91 \\
& 6 & $5.53 \mathrm{E}-09$ & 4.00 & $1.12 \mathrm{E}-20$ & 8.01 & $5.28 \mathrm{E}-20$ & 8.00 \\
& 7 & $3.45 \mathrm{E}-10$ & 4.00 & $4.37 \mathrm{E}-23$ & 8.00 & $2.06 \mathrm{E}-22$ & 8.00 \\
\hline
\end{tabular}



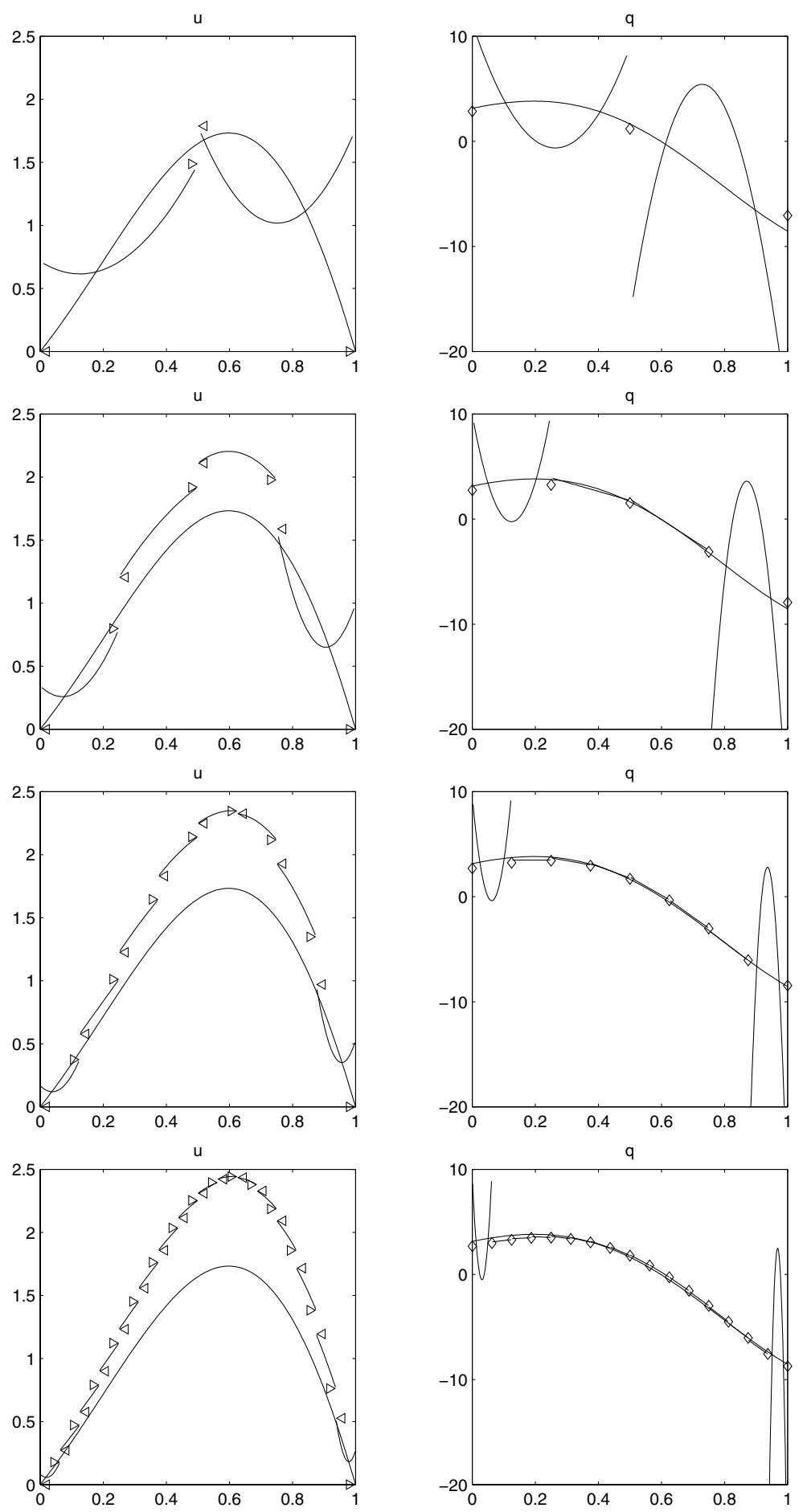

Figure 1. The B.Z. method with $\alpha=p / h$ and $p=2$. 

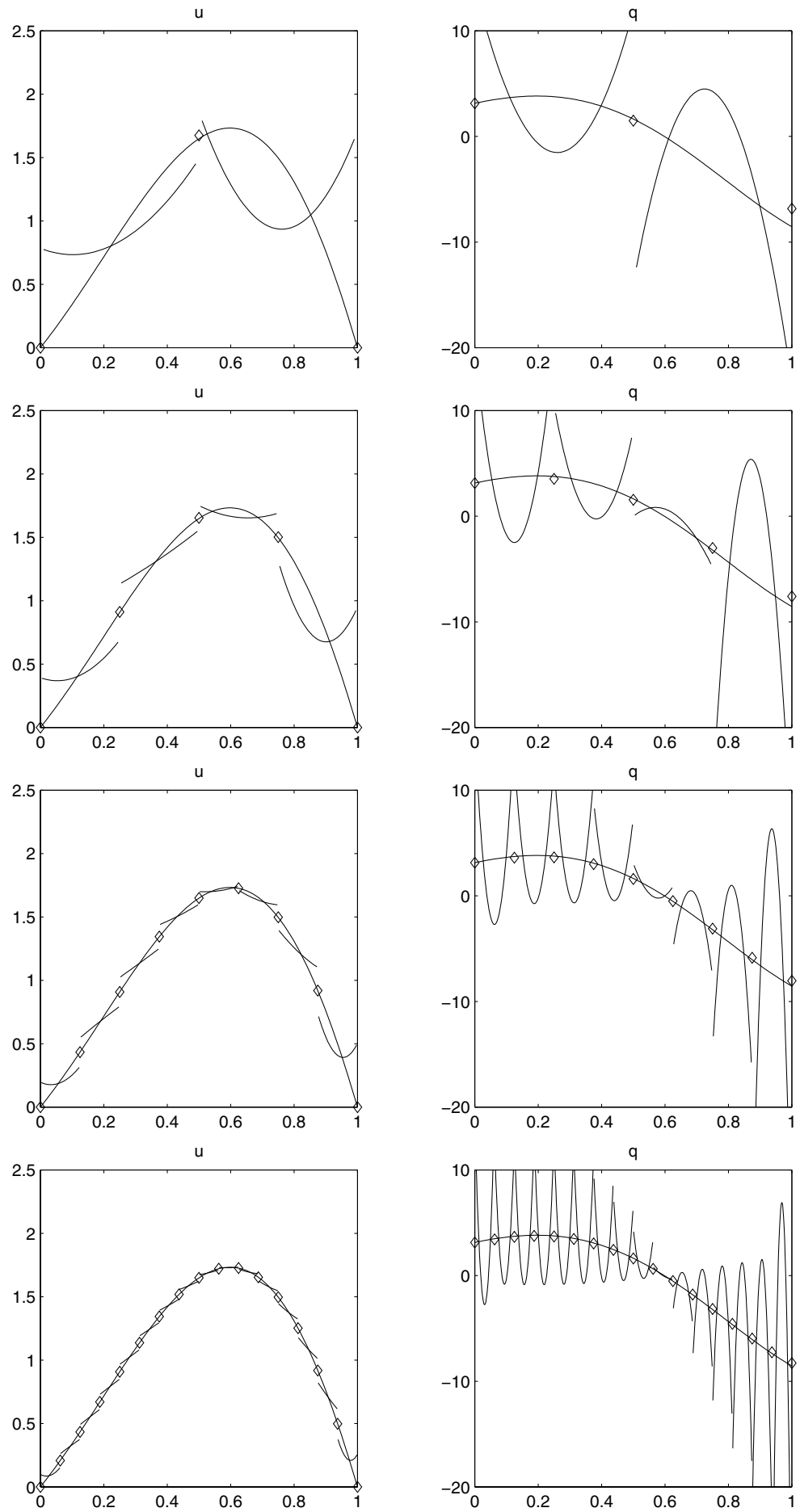

Figure 2. The m-B.Z. method with $\alpha=p / h$ and $p=2$. 
TABLE 10. History of convergence of the md-LDG method.

\begin{tabular}{cccccccc}
\hline & \multicolumn{4}{c}{$\left\|\left(e_{u}, e_{q}\right)\right\|_{c, \Omega_{h}}$} & \multicolumn{2}{c}{$\| \widehat{e_{u}} \epsilon_{\infty}$} & \multicolumn{2}{c}{$\left\|\widehat{e_{q}}-c{\widehat{e_{u}}}^{c}\right\|_{\infty}$} \\
$\mathrm{p}$ m mesh & error & order & error & order & error & order \\
\hline \hline \multirow{2}{*}{1} & 4 & $1.26 \mathrm{E}-02$ & 2.00 & $3.59 \mathrm{E}-05$ & 2.95 & $9.76 \mathrm{E}-05$ & 3.00 \\
& 5 & $3.15 \mathrm{E}-03$ & 2.00 & $4.62 \mathrm{E}-06$ & 2.96 & $1.22 \mathrm{E}-05$ & 3.00 \\
& 6 & $7.88 \mathrm{E}-04$ & 2.00 & $5.84 \mathrm{E}-07$ & 2.99 & $1.53 \mathrm{E}-06$ & 3.00 \\
& 7 & $1.97 \mathrm{E}-04$ & 2.00 & $7.33 \mathrm{E}-08$ & 2.99 & $1.91 \mathrm{E}-07$ & 3.00 \\
\hline & 4 & $2.42 \mathrm{E}-04$ & 2.98 & $8.51 \mathrm{E}-09$ & 4.96 & $4.98 \mathrm{E}-09$ & 4.93 \\
2 & 5 & $3.04 \mathrm{E}-05$ & 2.99 & $2.67 \mathrm{E}-10$ & 5.00 & $1.59 \mathrm{E}-10$ & 4.97 \\
& 6 & $3.81 \mathrm{E}-06$ & 3.00 & $8.35 \mathrm{E}-12$ & 5.00 & $5.04 \mathrm{E}-12$ & 4.98 \\
& 7 & $4.76 \mathrm{E}-07$ & 3.00 & $2.61 \mathrm{E}-13$ & 5.00 & $1.59 \mathrm{E}-13$ & 4.99 \\
\hline & 4 & $2.88 \mathrm{E}-06$ & 3.99 & $1.73 \mathrm{E}-13$ & 6.99 & $1.03 \mathrm{E}-12$ & 6.99 \\
3 & 5 & $1.81 \mathrm{E}-07$ & 4.00 & $1.34 \mathrm{E}-15$ & 7.01 & $8.06 \mathrm{E}-15$ & 7.00 \\
& 6 & $1.13 \mathrm{E}-08$ & 4.00 & $1.04 \mathrm{E}-17$ & 7.01 & $6.31 \mathrm{E}-17$ & 7.00 \\
& 7 & $7.06 \mathrm{E}-10$ & 4.00 & $8.12 \mathrm{E}-20$ & 7.00 & $4.93 \mathrm{E}-19$ & 7.00 \\
\hline & 4 & $2.86 \mathrm{E}-08$ & 4.99 & $2.08 \mathrm{E}-17$ & 8.99 & $1.16 \mathrm{E}-17$ & 9.04 \\
4 & 5 & $8.95 \mathrm{E}-10$ & 5.00 & $4.08 \mathrm{E}-20$ & 8.99 & $2.24 \mathrm{E}-20$ & 9.02 \\
& 6 & $2.80 \mathrm{E}-11$ & 5.00 & $7.99 \mathrm{E}-23$ & 9.00 & $4.34 \mathrm{E}-23$ & 9.01 \\
& 7 & $8.76 \mathrm{E}-13$ & 5.00 & $1.57 \mathrm{E}-25$ & 9.00 & $8.52 \mathrm{E}-26$ & 8.99 \\
\hline
\end{tabular}

TABLE 11. History of convergence of the h-R.T. method.

\begin{tabular}{cccccccc}
\hline & \multicolumn{3}{c}{$\left\|\left(e_{u}, e_{q}\right)\right\|_{c, \Omega_{h}}$} & \multicolumn{2}{c}{$\left\|{\widehat{e_{u}}}^{\epsilon}\right\|_{\infty}$} & \multicolumn{2}{c}{$\left\|\widehat{e_{q}}-c{\widehat{e_{u}}}^{c}\right\|_{\infty}$} \\
\multicolumn{1}{c}{ p mesh } & error & order & error & order & error & order \\
\hline \hline \multirow{2}{*}{1} & 4 & $4.42 \mathrm{E}-03$ & 2.00 & $1.37 \mathrm{E}-06$ & 4.12 & $2.86 \mathrm{E}-06$ & 4.01 \\
& 5 & $1.11 \mathrm{E}-03$ & 1.99 & $8.16 \mathrm{E}-08$ & 4.07 & $1.78 \mathrm{E}-07$ & 4.01 \\
& 6 & $2.78 \mathrm{E}-04$ & 2.00 & $4.96 \mathrm{E}-09$ & 4.04 & $1.11 \mathrm{E}-08$ & 4.00 \\
& 7 & $6.95 \mathrm{E}-05$ & 2.00 & $3.06 \mathrm{E}-10$ & 4.02 & $6.94 \mathrm{E}-10$ & 4.00 \\
\hline \multirow{3}{*}{2} & 4 & $6.37 \mathrm{E}-05$ & 3.01 & $1.25 \mathrm{E}-09$ & 4.77 & $4.01 \mathrm{E}-09$ & 5.06 \\
& 5 & $7.94 \mathrm{E}-06$ & 3.00 & $4.24 \mathrm{E}-11$ & 4.88 & $1.22 \mathrm{E}-10$ & 5.03 \\
& 6 & $9.92 \mathrm{E}-07$ & 3.00 & $1.38 \mathrm{E}-12$ & 4.94 & $3.78 \mathrm{E}-12$ & 5.02 \\
& 7 & $1.24 \mathrm{E}-07$ & 3.00 & $4.35 \mathrm{E}-14$ & 4.99 & $1.18 \mathrm{E}-13$ & 5.00 \\
\hline \multirow{3}{*}{3} & 4 & $9.88 \mathrm{E}-07$ & 3.97 & $1.69 \mathrm{E}-14$ & 8.05 & $1.40 \mathrm{E}-14$ & 7.94 \\
& 5 & $6.25 \mathrm{E}-08$ & 3.98 & $6.47 \mathrm{E}-17$ & 8.03 & $5.59 \mathrm{E}-17$ & 7.97 \\
& 6 & $3.93 \mathrm{E}-09$ & 3.99 & $2.50 \mathrm{E}-19$ & 8.02 & $2.20 \mathrm{E}-19$ & 7.99 \\
& 7 & $2.46 \mathrm{E}-10$ & 4.00 & $9.76 \mathrm{E}-22$ & 8.00 & $8.60 \mathrm{E}-22$ & 8.00 \\
\hline & 4 & $9.44 \mathrm{E}-09$ & 5.02 & $2.48 \mathrm{E}-18$ & 9.20 & $1.24 \mathrm{E}-17$ & 8.99 \\
4 & 5 & $2.93 \mathrm{E}-10$ & 5.01 & $4.45 \mathrm{E}-21$ & 9.12 & $2.43 \mathrm{E}-20$ & 8.99 \\
& 6 & $9.12 \mathrm{E}-12$ & 5.01 & $8.27 \mathrm{E}-24$ & 9.07 & $4.75 \mathrm{E}-23$ & 9.00 \\
& 7 & $2.85 \mathrm{E}-13$ & 5.00 & $1.58 \mathrm{E}-26$ & 9.03 & $9.29 \mathrm{E}-26$ & 9.00 \\
\hline
\end{tabular}

since the method does not diverge, the orders of convergence of its numerical traces are at least $p$, in full agreement with Theorem 2.1. An illustration of this behavior is displayed in Figure 2. Comparing this figure with Figure 1 for the original B.Z. method, we can see the dramatic improvement on the quality of the approximation in the numerical traces induced by having a conservative numerical trace.

In Table 9 we display our numerical results for the m-B.Z. method for $\alpha=$ $(p / h)^{p+1}$. We observe that a convergence of order $p$ in the $\|\cdot\|_{c, \Omega_{h}}$-norm is obtained and that, in agreement with Theorem 2.1, the orders of converge of the numerical traces are $2 p$.

Finally, in Tables 10 and 11 we display the numerical results for md-LDG and h-R.T. methods. We see that both numerical traces converge with order $2 p+1$ as predicted by Theorem 2.1 .

4.3. Postprocessing of methods with conservative numerical traces. In Table 12, we see that the postprocessed solution $\left(q_{h}^{*}, u_{h}^{*}\right)$ of the md-LDG method 
TABLE 12. History of convergence of the postprocessed md-LDG method.

\begin{tabular}{cccccc}
\hline & \multicolumn{4}{c}{$\left\|u-u_{h}^{*}\right\|_{\infty, \Omega_{h}}$} & \multicolumn{2}{c}{$\left\|q-q_{h}^{*}\right\|_{\infty, \Omega_{h}}$} \\
$\mathrm{p}$ mesh & error & order & error & order \\
\hline \hline & 4 & $2.09 \mathrm{E}-04$ & 2.96 & $1.26 \mathrm{E}-03$ & 2.98 \\
1 & 5 & $2.64 \mathrm{E}-05$ & 2.98 & $1.58 \mathrm{E}-04$ & 2.99 \\
& 6 & $3.37 \mathrm{E}-06$ & 2.97 & $1.98 \mathrm{E}-05$ & 3.00 \\
& 7 & $4.33 \mathrm{E}-07$ & 2.96 & $2.48 \mathrm{E}-06$ & 3.00 \\
\hline & 4 & $4.52 \mathrm{E}-08$ & 4.99 & $1.67 \mathrm{E}-07$ & 4.88 \\
2 & 5 & $1.42 \mathrm{E}-09$ & 5.00 & $5.41 \mathrm{E}-09$ & 4.94 \\
& 6 & $4.42 \mathrm{E}-11$ & 5.00 & $1.72 \mathrm{E}-10$ & 4.97 \\
& 7 & $1.38 \mathrm{E}-12$ & 5.00 & $5.43 \mathrm{E}-12$ & 4.99 \\
\hline & 4 & $2.85 \mathrm{E}-12$ & 6.99 & $7.55 \mathrm{E}-12$ & 6.98 \\
3 & 5 & $2.22 \mathrm{E}-14$ & 7.00 & $5.90 \mathrm{E}-14$ & 7.00 \\
& 6 & $1.73 \mathrm{E}-16$ & 7.00 & $4.61 \mathrm{E}-16$ & 7.00 \\
& 7 & $1.35 \mathrm{E}-18$ & 7.00 & $3.60 \mathrm{E}-18$ & 7.00 \\
\hline & 4 & $1.12 \mathrm{E}-16$ & 8.92 & $2.53 \mathrm{E}-16$ & 9.01 \\
4 & 5 & $2.25 \mathrm{E}-19$ & 8.96 & $4.94 \mathrm{E}-19$ & 9.00 \\
& 6 & $4.45 \mathrm{E}-22$ & 8.98 & $9.63 \mathrm{E}-22$ & 9.00 \\
& 7 & $8.75 \mathrm{E}-25$ & 8.99 & $1.88 \mathrm{E}-24$ & 9.00 \\
\hline
\end{tabular}

converges uniformly in the whole domain with order $2 p+1$, as predicted by Proposition 2.3 .

4.4. The effect of negative "stabilization" parameters $\alpha$. We end our numerical experiments by displaying the performance of the LDG method with $\beta=0$ and a negative stabilization parameter $\alpha=-\frac{2(2 p+1)}{h}$. This DG method is well defined since the condition $\alpha<-\frac{3(2 p+1)}{2 h}$ is satisfied; see [16]. In Table 13, we display the history of convergence of the method. We see that the method converges with order $p$ in the $\|(\cdot, \cdot)\|_{c, \Omega_{h}}$-norm and that its numerical traces converge with order $2 p$; this behavior agrees with Theorem 2.1. In Table 14, we compare the errors of the method to those obtained with the positive stabilization parameter $\alpha=\frac{2(2 p+1)}{h}$. We see that they are not significantly different, although it is clear that those with a positive stabilization parameter are better, especially for coarser

TABLE 13. History of convergence of the LDG method with $\beta=0$ and $\alpha=-\frac{2(2 p+1)}{h}$.

\begin{tabular}{cccccccc}
\hline & \multicolumn{4}{c}{$\left\|\left(e_{u}, e_{q}\right)\right\|_{c, \Omega_{h}}$} & \multicolumn{2}{c}{$\left\|\widehat{e_{u}}\right\|_{\infty}$} & \multicolumn{2}{c}{$\left\|\widehat{e_{q}}-c \widehat{e_{u}}{ }^{c}\right\|_{\infty}$} \\
$\mathrm{p}$ mesh & error & order & error & order & error & order \\
\hline \hline & 4 & $3.37 \mathrm{E}-01$ & 1.25 & $1.71 \mathrm{E}-03$ & 2.23 & $2.94 \mathrm{E}-03$ & 2.05 \\
1 & 5 & $1.53 \mathrm{E}-01$ & 1.14 & $3.94 \mathrm{E}-04$ & 2.12 & $7.29 \mathrm{E}-04$ & 2.01 \\
& 6 & $7.28 \mathrm{E}-02$ & 1.07 & $9.45 \mathrm{E}-05$ & 2.06 & $1.81 \mathrm{E}-04$ & 2.00 \\
& 7 & $3.55 \mathrm{E}-02$ & 1.04 & $2.31 \mathrm{E}-05$ & 2.03 & $4.54 \mathrm{E}-05$ & 2.00 \\
\hline & 4 & $1.24 \mathrm{E}-02$ & 2.05 & $5.55 \mathrm{E}-07$ & 4.12 & $1.25 \mathrm{E}-06$ & 4.09 \\
2 & 5 & $2.97 \mathrm{E}-03$ & 2.06 & $3.24 \mathrm{E}-08$ & 4.10 & $7.68 \mathrm{E}-08$ & 4.02 \\
& 6 & $7.19 \mathrm{E}-04$ & 2.05 & $1.93 \mathrm{E}-09$ & 4.06 & $4.78 \mathrm{E}-09$ & 4.00 \\
& 7 & $1.76 \mathrm{E}-04$ & 2.03 & $1.18 \mathrm{E}-10$ & 4.04 & $2.99 \mathrm{E}-10$ & 4.00 \\
\hline & 4 & $1.86 \mathrm{E}-04$ & 3.32 & $4.73 \mathrm{E}-11$ & 6.28 & $2.36 \mathrm{E}-11$ & 6.05 \\
3 & 5 & $1.99 \mathrm{E}-05$ & 3.22 & $6.67 \mathrm{E}-13$ & 6.15 & $3.66 \mathrm{E}-13$ & 6.01 \\
& 6 & $2.26 \mathrm{E}-06$ & 3.14 & $9.88 \mathrm{E}-15$ & 6.08 & $5.70 \mathrm{E}-15$ & 6.00 \\
& 7 & $2.67 \mathrm{E}-07$ & 3.08 & $1.50 \mathrm{E}-16$ & 6.04 & $8.91 \mathrm{E}-17$ & 6.00 \\
\hline \multirow{3}{*}{4} & 4 & $2.82 \mathrm{E}-06$ & 4.04 & $1.13 \mathrm{E}-15$ & 7.83 & $7.3743 \mathrm{E}-15$ & 8.10 \\
& 5 & $1.76 \mathrm{E}-07$ & 4.00 & $4.54 \mathrm{E}-18$ & 7.95 & $2.8274 \mathrm{E}-17$ & 8.03 \\
& 6 & $1.10 \mathrm{E}-08$ & 4.00 & $1.79 \mathrm{E}-20$ & 7.99 & $1.0990 \mathrm{E}-19$ & 8.01 \\
& 7 & $6.87 \mathrm{E}-10$ & 4.00 & $7.02 \mathrm{E}-23$ & 8.00 & $4.3038 \mathrm{E}-22$ & 8.00 \\
\hline
\end{tabular}


TABLE 14. The LDG method with $\beta=0$ : Ratio of the errors with $\alpha=-\frac{2(2 p+1)}{h}$ to the errors with $\alpha=+\frac{2(2 p+1)}{h}$.

\begin{tabular}{|c|c|c|c|c|}
\hline $\mathrm{p}$ & mesh & $\begin{array}{c}\left\|\left(e_{u}, e_{q}\right)\right\|_{c, \Omega_{h}} \\
\text { ratio }\end{array}$ & $\begin{array}{l}\left\|{\widehat{e_{u}}}^{\epsilon}\right\|_{\infty} \\
\text { ratio }\end{array}$ & $\begin{array}{c}\left\|\widehat{e_{q}}-c{\widehat{e_{u}}}^{c}\right\|_{\infty} \\
\text { ratio }\end{array}$ \\
\hline \multirow{4}{*}{1} & 4 & 1.24 & 1.24 & 1.01 \\
\hline & 5 & 1.12 & 1.12 & 1.00 \\
\hline & 6 & 1.05 & 1.06 & 0.99 \\
\hline & 7 & 1.03 & 1.03 & 1.00 \\
\hline \multirow{4}{*}{2} & 4 & 3.40 & 3.65 & 3.07 \\
\hline & 5 & 3.25 & 3.35 & 3.01 \\
\hline & 6 & 3.14 & 3.17 & 3.01 \\
\hline & 7 & 3.08 & 3.09 & 3.01 \\
\hline \multirow{4}{*}{3} & 4 & 1.55 & 1.33 & 1.01 \\
\hline & 5 & 1.28 & 1.15 & 1.00 \\
\hline & 6 & 1.14 & 1.08 & 1.00 \\
\hline & 7 & 1.07 & 1.04 & 1.00 \\
\hline \multirow{4}{*}{4} & 4 & 2.98 & 2.84 & 3.08 \\
\hline & 5 & 2.99 & 2.95 & 3.02 \\
\hline & 6 & 3.00 & 2.98 & 3.00 \\
\hline & 7 & 3.00 & 3.00 & 3.00 \\
\hline
\end{tabular}

meshes and even values of $p$. In other words, this means that "stabilizing" with a negative parameter can have the same effect as "stabilizing" with a positive one.

4.5. An exact solution with a singularity. We end our numerical experiments by exploring a situation not covered by our main result. In Table 15, we display the approximation properties of the md-LDG method for the exact solution $u(x)=x^{7 / 2}$. Due to the lack of smoothness of the exact solution, the error $\left\|\left(e_{u}, e_{q}\right)\right\|_{c, \Omega_{h}}$ converges only with order $\min \{p+1,3\}$ (strictly speaking, with order $\min \{p+1,3-\delta\}$, for all $\delta>0$ ), no matter how high the polynomial degree is. Theorem 2.1 predicts that the numerical traces should converge with order $\min \{p+1,3\}+p$. This actually happens for $p=1$ and $p=2$, but for not for $p \geq 3$. In the latter case, we see convergence of order $\min \{p+1,3\}+p+1 / 2$.

TABLE 15. History of convergence of the md-LDG method for $u(x)=x^{7 / 2}$.

\begin{tabular}{cccccccc}
\hline & \multicolumn{4}{c}{$\left\|\left(e_{u}, e_{q}\right)\right\|_{c, \Omega_{h}}$} & \multicolumn{2}{c}{$\left\|\widehat{e_{u}}\right\|_{\infty}$} & \multicolumn{2}{c}{$\left\|\widehat{e_{q}}-c \widehat{e_{u}}{ }^{c}\right\|_{\infty}$} \\
$\mathrm{p}$ p mesh & error & order & error & order & error & order \\
\hline \hline \multirow{2}{*}{1} & 4 & $3.19 \mathrm{E}-03$ & 1.96 & $4.38 \mathrm{E}-06$ & 2.99 & $2.61 \mathrm{E}-05$ & 2.97 \\
& 5 & $8.06 \mathrm{E}-04$ & 1.99 & $5.48 \mathrm{E}-07$ & 3.00 & $3.30 \mathrm{E}-06$ & 2.99 \\
& 6 & $2.02 \mathrm{E}-04$ & 1.99 & $6.86 \mathrm{E}-08$ & 3.00 & $4.15 \mathrm{E}-07$ & 2.99 \\
& 7 & $5.07 \mathrm{E}-05$ & 2.00 & $8.57 \mathrm{E}-09$ & 3.00 & $5.20 \mathrm{E}-08$ & 3.00 \\
\hline & 4 & $2.90 \mathrm{E}-05$ & 2.93 & $3.90 \mathrm{E}-10$ & 4.87 & $1.93 \mathrm{E}-09$ & 4.95 \\
2 & 5 & $3.77 \mathrm{E}-06$ & 2.94 & $1.28 \mathrm{E}-11$ & 4.93 & $6.17 \mathrm{E}-11$ & 4.97 \\
& 6 & $4.89 \mathrm{E}-07$ & 2.95 & $4.12 \mathrm{E}-13$ & 4.96 & $1.95 \mathrm{E}-12$ & 4.98 \\
& 7 & $6.31 \mathrm{E}-08$ & 2.95 & $1.31 \mathrm{E}-14$ & 4.97 & $6.16 \mathrm{E}-14$ & 4.99 \\
\hline & 4 & $1.12 \mathrm{E}-06$ & 3.07 & $1.59 \mathrm{E}-13$ & 6.27 & $3.18 \mathrm{E}-13$ & 6.39 \\
3 & 5 & $1.36 \mathrm{E}-07$ & 3.04 & $1.95 \mathrm{E}-15$ & 6.35 & $3.69 \mathrm{E}-15$ & 6.43 \\
& 6 & $1.67 \mathrm{E}-08$ & 3.02 & $2.32 \mathrm{E}-17$ & 6.40 & $4.22 \mathrm{E}-17$ & 6.45 \\
& 7 & $2.07 \mathrm{E}-09$ & 3.01 & $2.69 \mathrm{E}-19$ & 6.43 & $4.77 \mathrm{E}-19$ & 6.47 \\
\hline & 4 & $2.25 \mathrm{E}-07$ & 3.02 & $1.33 \mathrm{E}-16$ & 7.38 & $2.28 \mathrm{E}-16$ & 7.44 \\
4 & 5 & $2.80 \mathrm{E}-08$ & 3.01 & $7.70 \mathrm{E}-19$ & 7.43 & $1.29 \mathrm{E}-18$ & 7.47 \\
& 6 & $3.49 \mathrm{E}-09$ & 3.00 & $4.38 \mathrm{E}-21$ & 7.46 & $7.17 \mathrm{E}-21$ & 7.49 \\
& 7 & $4.35 \mathrm{E}-10$ & 3.00 & $2.46 \mathrm{E}-23$ & 7.48 & $3.98 \mathrm{E}-23$ & 7.49 \\
\hline
\end{tabular}


An explanation of the appearance of the additional term $1 / 2$ can be obtained by a simple modification of the proof of Theorem 2.1. The idea is as follows: since the main part of the error is concentrated around the singularity, that is, around $x=0$, it suffices to estimate the error in that region in a different way. Let us briefly sketch how to do this for $\widehat{e_{u}}\left(x_{i}\right)$. We assume, of course, that $p \geq 3$.

Our starting point is again the error representation formula for $\widehat{e_{u}}\left(x_{i}\right)$ of Lemma 3.1, which for the md-LDG method can take the particular form given by Lemma 3.3. We only show how to estimate one of the terms in such a formula, namely, $T:=\left(q-\pi^{+} q,\left(\pi^{+} \varphi_{x_{i}}-\varphi_{x_{i}}\right)^{\prime}\right)_{\Omega_{h}}$. We estimate it as follows. First, we rewrite it as $T=T_{1}+T_{2}$, where

$$
T_{1}=\left(q-\pi^{+} q,\left(\pi^{+} \varphi_{x_{i}}-\varphi_{x_{i}}\right)^{\prime}\right)_{I_{1}}
$$

Then,

$$
\begin{aligned}
\left|T_{1}\right| & \leq\left\|q-\pi^{+} q\right\|_{0, I_{1}}\left\|\left(\pi^{+} \varphi_{x_{i}}-\varphi_{x_{i}}\right)^{\prime}\right\|_{0, I_{1}} \\
& \leq\left\|q-\pi^{+} q\right\|_{0, I_{1}}\left\|\left(\pi^{+} \varphi_{x_{i}}-\varphi_{x_{i}}\right)^{\prime}\right\|_{L^{\infty}\left(I_{1}\right)} h^{1 / 2} \\
& \leq C_{\delta} h^{3-\delta+p+1 / 2}\left\|\varphi_{x_{i}}^{(p+1)}\right\|_{L^{\infty}\left(I_{1}\right)}
\end{aligned}
$$

since $q=\frac{7}{2} \epsilon x^{5 / 2}$, and

$$
\begin{aligned}
\left|T_{2}\right| & \leq\left\|q-\pi^{+} q\right\|_{0, \Omega_{h} \backslash I_{1}}\left\|\left(\pi^{+} \varphi_{x_{i}}-\varphi_{x_{i}}\right)^{\prime}\right\|_{0, \Omega_{h} \backslash I_{1}} \\
& \leq\left\|q-\pi^{+} q\right\|_{0, \Omega_{h} \backslash I_{1}} h^{p+1}\left|\varphi_{x_{i}}\right|_{p+1, \Omega_{h}} .
\end{aligned}
$$

Exploiting the form of the singularity for $q$, we get

$$
\left|T_{2}\right| \leq C_{\delta} h^{3-\delta+p+1 / 2}\left|\varphi_{x_{i}}\right|_{p+1, \Omega_{h}} .
$$

This completes our sketch of the explanation of the extra term $1 / 2$.

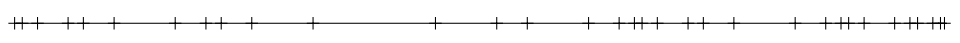

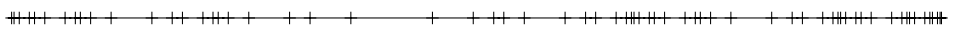

FIGURE 3. Nonuniform meshes. 
TABLE 16. History of convergence of the md-LDG method with nonuniform meshes.

\begin{tabular}{cccccccc}
\hline & \multicolumn{4}{c}{$\left\|\left(e_{u}, e_{q}\right)\right\|_{c, \Omega_{h}}$} & \multicolumn{2}{c}{$\left\|\widehat{e_{u}}\right\|_{\infty}$} & \multicolumn{2}{c}{$\left\|\widehat{e_{q}}-c \widehat{e_{u}}\right\|_{\infty}$} \\
$\mathrm{p}$ mesh & error & order & error & order & error & order \\
\hline \hline & 4 & $6.60 \mathrm{E}-02$ & 2.34 & $4.27 \mathrm{E}-04$ & 3.90 & $1.26 \mathrm{E}-03$ & 3.86 \\
1 & 5 & $2.40 \mathrm{E}-02$ & 2.49 & $9.41 \mathrm{E}-05$ & 3.73 & $2.73 \mathrm{E}-04$ & 3.77 \\
& 6 & $9.02 \mathrm{E}-03$ & 2.42 & $2.02 \mathrm{E}-05$ & 3.79 & $5.72 \mathrm{E}-05$ & 3.86 \\
& 7 & $3.31 \mathrm{E}-03$ & 2.47 & $4.32 \mathrm{E}-06$ & 3.80 & $1.21 \mathrm{E}-05$ & 3.82 \\
\hline \multirow{3}{*}{2} & 4 & $1.32 \mathrm{E}-03$ & 3.58 & $5.27 \mathrm{E}-08$ & 7.62 & $7.52 \mathrm{E}-08$ & 7.94 \\
& 5 & $3.20 \mathrm{E}-04$ & 3.51 & $5.73 \mathrm{E}-09$ & 5.47 & $4.38 \mathrm{E}-09$ & 7.01 \\
& 6 & $7.75 \mathrm{E}-05$ & 3.49 & $4.32 \mathrm{E}-10$ & 6.37 & $4.75 \mathrm{E}-10$ & 5.48 \\
& 7 & $1.87 \mathrm{E}-05$ & 3.50 & $3.00 \mathrm{E}-11$ & 6.58 & $5.06 \mathrm{E}-11$ & 7.01 \\
\hline & 4 & $1.19 \mathrm{E}-04$ & 4.22 & $3.61 \mathrm{E}-10$ & 7.80 & $9.63 \mathrm{E}-10$ & 7.81 \\
3 & 5 & $1.81 \mathrm{E}-05$ & 4.66 & $1.52 \mathrm{E}-11$ & 7.81 & $3.70 \mathrm{E}-11$ & 8.03 \\
& 6 & $3.07 \mathrm{E}-06$ & 4.38 & $6.47 \mathrm{E}-13$ & 7.79 & $1.50 \mathrm{E}-12$ & 7.92 \\
& 7 & $4.81 \mathrm{E}-07$ & 4.57 & $2.51 \mathrm{E}-14$ & 8.01 & $5.81 \mathrm{E}-14$ & 8.01 \\
\hline \multirow{3}{*}{4} & 4 & $3.13 \mathrm{E}-06$ & 5.68 & $7.96 \mathrm{E}-14$ & 10.52 & $2.08 \mathrm{E}-13$ & 10.52 \\
& 5 & $3.56 \mathrm{E}-07$ & 5.36 & $1.76 \mathrm{E}-15$ & 9.40 & $4.17 \mathrm{E}-15$ & 9.64 \\
& 6 & $3.69 \mathrm{E}-08$ & 5.59 & $2.86 \mathrm{E}-17$ & 10.16 & $6.56 \mathrm{E}-17$ & 10.24 \\
& 7 & $4.07 \mathrm{E}-09$ & 5.44 & $5.31 \mathrm{E}-19$ & 9.83 & $1.21 \mathrm{E}-18$ & 9.84 \\
\hline
\end{tabular}

4.6. Nonuniform meshes. For simplicity, we have used uniform meshes in all the previous experiments. Similar results, however do hold for nonuniform meshes. To illustrate this, we present some numerical results where the approximate solution is obtained by employing a nonuniform mesh. Again $f$ is chosen so that the exact solution is $u=e^{x} \sin (\pi x)$. A sequence of nonuniform meshes are formed in the following way. We start with a mesh with two elements $I_{1}=[0,2 / 3]$ and $I_{2}=$ $[2 / 3,1]$. The next mesh is a refinement of this one where we divide $I_{1}$ into two parts with ratio one-to-two, and $I_{2}$ with ratio of two-to-one, hence the second mesh consists of the following elements: $I_{1}=[0,2 / 9], I_{2}=[2 / 9,6 / 9], I_{3}=[6 / 9,8 / 9]$, and $I_{4}=[8 / 9,1]$. Each consequent mesh is obtained by refining every element in this manner, that is, dividing odd numbered elements in a ratio of one-to-two and the even numbered elements in a ratio of two-to-one. A picture of the first six of these meshes is shown in Figure 3.

TABLE 17. History of convergence of the h-R.T. method with nonuniform meshes.

\begin{tabular}{cccccccc}
\hline & \multicolumn{3}{c}{$\left\|\left(e_{u}, e_{q}\right)\right\|_{c, \Omega_{h}}$} & \multicolumn{2}{c}{$\left\|\widehat{e_{u}} \epsilon\right\|_{\infty}$} & \multicolumn{2}{c}{$\left\|\widehat{e_{q}}-c \widehat{e_{u}}{ }^{c}\right\|_{\infty}$} \\
$\mathrm{p}$ mesh & error & order & error & order & error & order \\
\hline \hline & 4 & $1.51 \mathrm{E}-01$ & 2.74 & $4.06 \mathrm{E}-04$ & 4.96 & $2.50 \mathrm{E}-04$ & 4.88 \\
1 & 5 & $6.09 \mathrm{E}-02$ & 2.25 & $1.05 \mathrm{E}-04$ & 3.34 & $3.33 \mathrm{E}-05$ & 4.97 \\
& 6 & $2.02 \mathrm{E}-02$ & 2.72 & $1.32 \mathrm{E}-05$ & 5.11 & $4.56 \mathrm{E}-06$ & 4.90 \\
& 7 & $7.87 \mathrm{E}-03$ & 2.33 & $3.57 \mathrm{E}-06$ & 3.22 & $5.99 \mathrm{E}-07$ & 5.01 \\
\hline & 4 & $3.15 \mathrm{E}-03$ & 2.21 & $1.92 \mathrm{E}-07$ & 5.65 & $3.13 \mathrm{E}-07$ & 6.85 \\
2 & 5 & $8.41 \mathrm{E}-04$ & 3.26 & $2.30 \mathrm{E}-08$ & 5.23 & $5.23 \mathrm{E}-08$ & 4.41 \\
& 6 & $2.99 \mathrm{E}-04$ & 2.55 & $2.50 \mathrm{E}-09$ & 5.47 & $4.83 \mathrm{E}-09$ & 5.88 \\
& 7 & $9.45 \mathrm{E}-05$ & 2.84 & $2.85 \mathrm{E}-10$ & 5.36 & $6.04 \mathrm{E}-10$ & 5.13 \\
\hline & 4 & $5.22 \mathrm{E}-04$ & 3.98 & $4.22 \mathrm{E}-10$ & 7.96 & $6.98 \mathrm{E}-10$ & 8.00 \\
3 & 5 & $9.25 \mathrm{E}-05$ & 4.27 & $1.81 \mathrm{E}-11$ & 7.76 & $2.37 \mathrm{E}-11$ & 8.34 \\
& 6 & $1.77 \mathrm{E}-05$ & 4.08 & $6.69 \mathrm{E}-13$ & 8.14 & $8.65 \mathrm{E}-13$ & 8.17 \\
& 7 & $3.20 \mathrm{E}-06$ & 4.22 & $2.81 \mathrm{E}-14$ & 7.82 & $2.96 \mathrm{E}-14$ & 8.33 \\
\hline \multirow{3}{*}{4} & 4 & $1.03 \mathrm{E}-05$ & 5.40 & $9.99 \mathrm{E}-14$ & 9.74 & $2.67 \mathrm{E}-13$ & 10.28 \\
& 5 & $1.60 \mathrm{E}-06$ & 4.60 & $2.08 \mathrm{E}-15$ & 9.55 & $5.56 \mathrm{E}-15$ & 9.55 \\
& 6 & $2.01 \mathrm{E}-07$ & 5.12 & $4.10 \mathrm{E}-17$ & 9.69 & $9.52 \mathrm{E}-17$ & 10.03 \\
& 7 & $7.01 \mathrm{E}-09$ & 4.84 & $6.12 \mathrm{E}-20$ & 9.39 & $1.38 \mathrm{E}-19$ & 9.43 \\
\hline
\end{tabular}


In Tables 16 and 17 we display the history of convergence for the md-LDG method and the h-R.T. method, respectively. We see that for both methods, both numerical traces converge with order $2 p+1$.

\section{EXTENSIONS AND CONCLUDING REMARKS}

In this paper, the unsuspected relevance of the conservativity of the numerical traces for its superconvergence has been uncovered. Perhaps the most striking illustration of this fact is the comparison of the behavior of the B.Z. method and that of its slight modification, the m-B.Z. method. Indeed, we have shown that the numerical traces of the m-B.Z. method with polynomial approximations of degree $p$ converge with order $p$ when the penalization parameter $\alpha$ is equal to $p / h$; this happens even though the numerical trace $\widehat{q}_{h}$ is not consistent. In contrast, the numerical traces of the closely related B.Z. method, whose numerical trace $\widehat{u}_{h}^{\epsilon}$ is not conservative, do not even converge for the same choice of spaces and penalization parameter.

Let us recall that, in [4], it was established that DG methods with conservative numerical traces are adjoint consistent. A direct consequence of this fact is that error representation formulas for linear functionals can be obtained with which their superconvergence can be established. Those formulas are similar to the ones obtained in Lemma 3.1 for the errors in the quantities $u$ and flx. Moreover, the fact that the conservativity of the numerical traces $\widehat{u}_{h}^{\epsilon}$ and $\left(-\widehat{q}_{h}+c \widehat{u}_{h}^{c}\right)$ implies that

$$
\mathrm{u}=\widehat{u}_{h}^{\epsilon} \quad \text { and } \quad \mathrm{flx}=\left(-\widehat{q}_{h}+c \widehat{u}_{h}^{c}\right)
$$

is certainly not a coincidence, since in such a case, the method is adjoint consistent.

To end, let us point out that, although we worked with a very simple convectiondiffusion equation, our main result, Theorem 2.1, can be extended to general convection-diffusion equations in a straightforward way. Moreover, Neumann and Robin boundary conditions can be easily dealt with. The approach can also be applied to other types of equations. In particular, it has been applied to the study of discontinuous Galerkin methods for Timoshenko beams [16].

\section{APPENDIX: An ANALYSIS OF THE MD-LDG AND MD-DG METHODS}

In this appendix, we prove the following result.

Proposition 5.1. For the $m d-L D G$ and $m d-D G$ methods, we have

$\sqrt{\epsilon}\left|\left(\pi^{+} e_{q}, \pi^{-} e_{u}\right)\right|_{\mathcal{A}_{h}} \leq\left(\left\|\pi^{+} q-q\right\|_{0, \Omega_{h}}^{2}+\left\langle\epsilon \gamma, \llbracket \pi^{+} q n \rrbracket^{2}\right\rangle_{\mathscr{E}_{h}}+\frac{\epsilon}{\alpha}\left|\left(\pi^{+} q-q\right)(1)\right|^{2}\right)^{1 / 2}$.

To prove this result, we need to introduce some notation. First, note that the approximate solution given by the md-LDG method satisfies

$$
\mathscr{A}_{h}\left(q_{h}, u_{h} ; v, w\right):=b_{h}(v, w) \quad \forall v, w \in X_{h}^{p},
$$

where

$$
\begin{aligned}
\mathscr{A}_{h}\left(q_{h}, u_{h} ; v, w\right):= & \left(q_{h}, v\right)_{\Omega_{h}}+\left(\epsilon u_{h}, v^{\prime}\right)_{\Omega_{h}}-\left\langle\epsilon \widehat{u}_{h}^{\epsilon}, v n\right\rangle_{\partial \Omega_{h} \backslash \partial \Omega} \\
& +\left(q_{h}-c u_{h}, w^{\prime}\right)_{\Omega_{h}}-\left\langle\widehat{q}_{h}-c \widehat{u}_{h}^{c}, w n\right\rangle_{\partial \Omega_{h} \backslash \partial \Omega} \\
& +q_{h}\left(0^{+}\right) w\left(0^{+}\right)-\left(q_{h}\left(1^{-}\right)-c u_{h}\left(1^{-}\right)-\alpha u_{h}\left(1^{-}\right)\right) w\left(1^{-}\right)
\end{aligned}
$$

and

$$
b_{h}(v, w):=(f, w)_{\Omega_{h}}-\epsilon u_{0} v\left(0^{+}\right)+\epsilon u_{1} v\left(1^{-}\right)+\alpha u_{1} w\left(1^{-}\right)+c u_{0} w\left(0^{+}\right) .
$$


As a simple consequence of the consistency of the numerical traces we obtain the so-called Galerkin orthogonality property,

$$
\mathscr{A}_{h}\left(e_{q}, e_{u} ; v, w\right)=0 \quad \forall v, w \in V_{h}^{p} .
$$

We are now ready to prove Proposition 5.1.

Proof of Proposition 5.1. By the Galerkin orthogonality property,

$$
\begin{aligned}
\left|\left(\pi^{+} e_{q}, \pi^{-} e_{u}\right)\right|_{\mathscr{A}_{h}}^{2} & =\mathscr{A}_{h}\left(\pi^{+} e_{q}, \pi^{-} e_{u} ; \frac{1}{\epsilon} \pi^{+} e_{q}, \pi^{-} e_{u}\right) \\
& =\mathscr{A}_{h}\left(\pi^{+} q-q, \pi^{-} u-u ; \frac{1}{\epsilon} \pi^{+} e_{q}, \pi^{-} e_{u}\right),
\end{aligned}
$$

and by the properties of the projections $\pi^{ \pm}$and the definition of the bilinear form $\mathcal{A}_{h}(\cdot, \cdot)$,

$$
\begin{aligned}
\left|\left(\pi^{+} e_{q}, \pi^{-} e_{u}\right)\right|_{\mathscr{A}_{h}}^{2}= & \frac{1}{\epsilon}\left(\pi^{+} q-q, \pi^{+} e_{q}\right)_{\Omega_{h}}+\left\langle\epsilon \gamma \llbracket \pi^{+} q n \rrbracket, \frac{1}{\epsilon} \llbracket \pi^{+} e_{q} n \rrbracket\right\rangle_{\mathscr{E}_{h}^{\circ}} \\
& -\left(\pi^{+} q-q\right)\left(1^{-}\right) \pi^{-} e_{u}(1) .
\end{aligned}
$$

Since, by definition of the seminorm $|\cdot| \mathcal{A}_{h},(2.10)$,

$$
\begin{aligned}
\left|\left(\pi^{+} e_{q}, \pi^{-} e_{u}\right)\right|_{\mathcal{A}_{h}}^{2}= & \frac{1}{\epsilon}\left\|\pi^{+} e_{q}\right\|_{0, \Omega_{h}}^{2}+\frac{1}{2}\left\langle c, \llbracket \pi^{-} e_{u} n \rrbracket^{2}\right\rangle_{\mathscr{E}_{h}} \\
& +\left\langle\gamma, \llbracket \pi^{+} e_{q} n \rrbracket^{2}\right\rangle_{\mathscr{E}_{h}^{\circ}}+\alpha \pi^{-} e_{u}^{2}\left(1^{-}\right),
\end{aligned}
$$

a simple application of Cauchy's inequality gives

$$
\left|\left(\pi^{+} e_{q}, \pi^{-} e_{u}\right)\right|_{\mathscr{A}_{h}}^{2} \leq\left|\left(\pi^{+} e_{q}, \pi^{-} e_{u}\right)\right|_{\mathscr{A}_{h}} \Theta
$$

where

$$
\Theta=\frac{1}{\sqrt{\epsilon}}\left(\left\|\pi^{+} q-q\right\|_{0, \Omega_{h}}^{2}+\left\langle\epsilon \gamma, \llbracket \pi^{+} e_{q} n \rrbracket^{2}\right\rangle_{\mathscr{E}_{h}^{\circ}}+\frac{\epsilon}{\alpha}\left|\left(\pi^{+} q-q\right)(1)\right|^{2}\right)^{1 / 2}
$$

This completes the proof of Proposition 5.1.

\section{REFERENCES}

[1] S. Adjerid and T.C. Massey, Superconvergence of discontinuous Galerkin finite element solutions for transient convection-diffusion problems, J. Sci. Comput. 22 and 23 (2005), 5-24. MR2142188 (2006a:65120)

[2] D. N. Arnold, An interior penalty finite element method with discontinuous elements, SIAM J. Numer. Anal. 19 (1982), 742-760. MR0664882 (83f:65173)

[3] D. N. Arnold and F. Brezzi, Mixed and nonconforming finite element methods: implementation, postprocessing and error estimates, RAIRO Modél. Math. Anal. Numér. 19 (1985), 7-32. MR0813687 (87g:65126)

[4] D. N. Arnold, F. Brezzi, B. Cockburn, and L. D. Marini, Unified analysis of discontinuous Galerkin methods for elliptic problems, SIAM J. Numer. Anal. 39 (2002), 1749-1779. MR1885715 (2002k:65183)

[5] I. Babuška and M. Zlámal, Nonconforming elements in the finite element method with penalty, SIAM J. Numer. Anal. 10 (1973), 863-875. MR0345432 (49:10168)

[6] D. H. Bailey, A Fortran-90 Based Multiprecision System. ACM Transactions on Mathematical Software 21, no. 4 (1995), 379-387.

[7] G. A. Baker, Finite element methods for elliptic equations using nonconforming elements, Math. Comp. 31 (1977), 45-59. MR0431742 (55:4737)

[8] F. Bassi, S. Rebay, G. Mariotti, S. Pedinotti, and M. Savini, A high-order accurate discontinuous finite element method for inviscid and viscous turbomachinery flows, 2nd European Conference on Turbomachinery Fluid Dynamics and Thermodynamics (Antwerpen, Belgium) (R. Decuypere and G. Dibelius, eds.), Technologisch Instituut, March 5-7 1997, pp. 99-108. 
[9] C. E. Baumann and J. T. Oden, A discontinuous hp-finite element method for convectiondiffusion problems, Comput. Methods Appl. Mech. Engrg. 175 (1999), 311-341. MR1702201 (2000d:65171)

[10] C.L. Bottasso, S. Micheletti, and R. Sacco, The discontinuous Petrov-Galerkin method for elliptic problems, Comput. Methods Appl. Mech. Engrg. 191 (2002), 3391-3409. MR1908187 (2003h:65153)

[11] F. Brezzi, G. Manzini, L. D. Marini, P. Pietra, and A. Russo, Discontinuous finite elements for diffusion problems, in Atti Convegno in onore di F. Brioschi (Milan 1997), Istituto Lombardo, Accademia di Scienze e Lettere, 1999, pp. 197-217.

[12] _ Discontinuous Galerkin approximations for elliptic problems, Numer. Methods Partial Differential Equations 16 (2000), 365-378. MR1765651 (2001e:65178)

[13] P. Castillo, A superconvergence result for discontinuous Galerkin methods applied to elliptic problems, Comput. Methods Appl. Mech. Engrg. 192 (2003), 4675-4685. MR2012484 (2004k:65213)

[14] P. Castillo, B. Cockburn, I. Perugia, and D. Schötzau, An a priori error analysis of the local discontinuous Galerkin method for elliptic problems, SIAM J. Numer. Anal. 38 (2000), 1676-1706. MR1813251 (2002k:65175)

[15] P. Castillo, B. Cockburn, D. Schötzau, and C. Schwab, Optimal a priori error estimates for the hp-version of the local discontinuous Galerkin method for convection-diffusion problems, Math. Comp. 71 (2002), 455-478. MR1885610 (2003e:65214)

[16] F. Celiker, Discontinuous Galerkin methods for Structural Mechanics, Ph.D. thesis, University of Minnesota, 2005.

[17] F. Celiker and B. Cockburn, Element-by-element post-processing of discontinuous Galerkin methods for Timoshenko beams, J. Sci. Comput., to appear.

[18] B. Cockburn and C.-W. Shu, The local discontinuous Galerkin method for time-dependent convection-diffusion systems, SIAM J. Numer. Anal. 35 (1998), 2440-2463. MR1655854 (99j:65163)

[19] M. Delfour, W. Hager, and F. Trochu, Discontinuous Galerkin methods for ordinary differential equations, Math. Comp. 36 (1981), 455-473. MR0606506 (82b:65066)

[20] J. Douglas, Jr. and T. Dupont, Galerkin approximations for the two point boundary value problem using continuous, piecewise polynomial spaces, Numer. Math. 22 (1974), 99-109. MR0362922 (50:15360)

[21] T. Dupont, A unified theory of superconvergence for Galerkin methods for two-point boundary problems, SIAM J. Numer. Anal. 13 (1976), 362-368. MR0408256 (53:12021)

[22] M. Larson and A.J. Niklasson, Analysis of a family of discontinuous Galerkin methods for elliptic problems: the one dimensional case, Numer. Math. 99 (2004), 113-130. MR2101786 (2005i:65188)

[23] P. Lesaint and P. A. Raviart, On a finite element method for solving the neutron transport equation, Mathematical Aspects of Finite Elements in Partial Differential Equations (C. de Boor, ed.), Academic Press, 1974, pp. 89-145. MR0658142 (58:31918)

[24] P. A. Raviart and J. M. Thomas, A mixed finite element method for second order elliptic problems, Mathematical Aspects of Finite Element Method (I. Galligani and E. Magenes, eds.), Lecture Notes in Math. 606, Springer-Verlag, New York, 1977, pp. 292-315. MR0483555 (58:3547)

[25] B. Rivière, M. F. Wheeler, and V. Girault, Improved energy estimates for interior penalty, constrained and discontinuous Galerkin methods for elliptic problems. Part I, Comp. Geosci. 3 (1999), 337-360. MR1750076 (2001d:65145)

[26] D. Schötzau, hp-DGFEM for parabolic evolution problems - applications to diffusion and viscous incompressible fluid flow, Ph.D. thesis, Swiss Federal Institute of Technology, Zürich, 1999.

[27] C. Schwab, p- and hp-finite element methods. Theory and applications to solid and fluid mechanics, Oxford Univ. Press, 1998. MR1695813 (2000d:65003)

[28] J. A. Wheeler, Simulation of heat transfer from a warm pipe buried in permafrost, 74th National Meeting of the American Institute of Chemical Engineers, New Orleans (March, 1973).

[29] M. F. Wheeler, A Galerkin procedure for estimating the flux for two-point boundary value problems, SIAM J. Numer. Anal. 11 (1974), 764-768. MR0383764 (52:4644) 
[30] Z. Zhang, Finite element superconvergence approximation for one-dimensional singularly perturbed problems, Numer. Methods Partial Differential Equations 18 (2002), 374-395. MR1895005 (2003c:65063)

[31] _ On the $h p$ finite element method for the one dimensional singularly perturbed convection-diffusion problems, J. Comput. Math. 20 (2002), no. 6, 599-610. MR1938640 (2003j:65071)

School of Mathematics, University of Minnesota, 206 Church Street S.E., MinneapoLis, Minnesota 55455

E-mail address: celiker@math.umn.edu

School of Mathematics, University of Minnesota, 206 Church Street S.E., MinneapoLis, Minnesota 55455

E-mail address: cockburn@math.umn.edu 